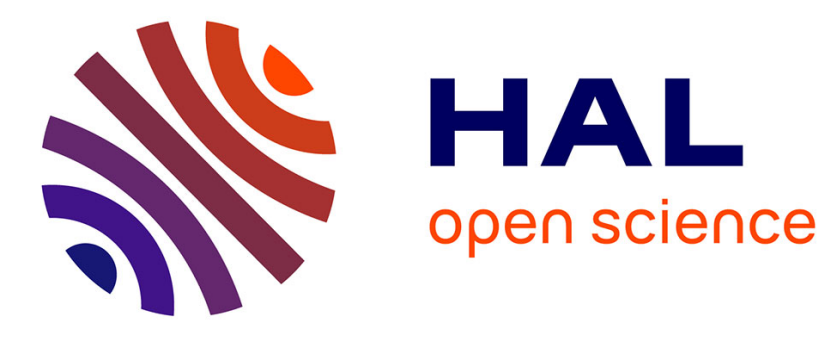

\title{
Opto nongenetics inhibition of neuronal firing
}

Karima Ait Ouares, Corinne Beurrier, Marco Canepari, Gwenaelle Laverne, Nicola Kuczewski

\section{To cite this version:}

Karima Ait Ouares, Corinne Beurrier, Marco Canepari, Gwenaelle Laverne, Nicola Kuczewski. Opto nongenetics inhibition of neuronal firing. European Journal of Neuroscience, 2019, 49 (1), pp.6-26. 10.1111/ejn.14251 . hal-01994645

\section{HAL Id: hal-01994645 \\ https://hal.science/hal-01994645}

Submitted on 28 Feb 2020

HAL is a multi-disciplinary open access archive for the deposit and dissemination of scientific research documents, whether they are published or not. The documents may come from teaching and research institutions in France or abroad, or from public or private research centers.
L'archive ouverte pluridisciplinaire HAL, est destinée au dépôt et à la diffusion de documents scientifiques de niveau recherche, publiés ou non, émanant des établissements d'enseignement et de recherche français ou étrangers, des laboratoires publics ou privés. 


\section{European Journal of Neuroscience}

\section{Opto non-genetics inhibition of neuronal firing}

\begin{tabular}{|r|l|}
\hline Journal: & European Journal of Neuroscience \\
\hline Manuscript ID & EJN-2018-08-25818.R1 \\
\hline Manuscript Type: & Registered Report (Stage 2) \\
\hline Date Submitted by the Author: & n/a \\
\hline Complete List of Authors: & $\begin{array}{l}\text { Ait Ouares, Karima; Université Grenoble Alpes et CNRS UMR 5588, } \\
\text { Laboratoire Interdisciplinaire de Physique (LIPhy).Laboratories of } \\
\text { Excellence, Ion Channel Science and Therapeutics, France } \\
\text { Beurrier, Corinne; UMR7288 (CNRS/Aix-Marseille Université), Institut de } \\
\text { Biologie du Développement de Marseille (IBDM) } \\
\text { Canepari, Marco; Université Grenoble Alpes et CNRS UMR 5588, } \\
\text { Laboratoire Interdisciplinaire de Physique (LIPhy), Laboratories of } \\
\text { Excellence, Ion Channel Science and Therapeutics, France.Institut National } \\
\text { de la Santé et Recherche Médicale, France } \\
\text { laverne, gwenaelle; UMR7288 (CNRS/Aix-Marseille Université), Institut de } \\
\text { Biologie du Développement de Marseille (IBDM) } \\
\text { Kuczewski, Nicola; Université Claude Bernard, Centre de Recherche en } \\
\text { neuroscience de Lyon }\end{array}$ \\
\hline Key Words: & \begin{tabular}{l} 
mice, optogenetics, brain slices, light stimulation \\
\hline
\end{tabular} \\
\hline
\end{tabular}




\section{Opto non-genetics inhibition of neuronal firing \\ K. Ait Ouares ${ }^{1 *}$, C. Beurrier ${ }^{2 *}$, M. Canepari ${ }^{1,3} *$ G.Laverne $^{2 *}$, N. Kuczewski $^{4}$ \\ (* equal contribution)}

${ }^{1}$ Univ. Grenoble Alpes, CNRS, LIPhy, F-38000 Grenoble, France. Laboratories of Excellence, Ion Channel Science and Therapeutics, France. ${ }^{2}$ Aix Marseille University, CNRS, IBDM, Marseille, France. ${ }^{3}$ Univ. Grenoble Alpes, CNRS, LIPhy, F-38000 Grenoble, France. Laboratories of Excellence, Ion Channel Science and Therapeutics, France.Institut National de la Santé et Recherche Médicale, France. ${ }^{3}$ CNRS, UMR 5292; INSERM, U1028; Lyon Neuroscience Research Center, Neuroplasticity and neuropathology of olfactory perception Team, Lyon, F-69000, France; University Lyon,

F-69000, France; University Lyon1, Villeurbanne, F-69000, France.

\section{Corresponding author:}

Nicola Kuczewski

Centre de Recherche en Neurosciences de Lyon

UMR CNRS 5292 INSERM 1028 Université Lyon1

50 Avenue Tony Garnier

F-69007 LYON, FRANCE

Tel: (+33) 437287462

Fax: (+33) 437287601

e-mail:nicola.kuczewski@univ-lyon1.fr

Running title: Neuronal sensitivity to visual light stimulation

Total number of pages:39

Total number of figures: 16

Total number of tables: 3

The total number of words in the whole manuscript: 12,775

The total number of words in the Abstract:192

Keywords: optogenetics, mice, olfactory bulb, cerebellum, hippocampus, striatum. 


\begin{abstract}
Optogenetics is based on the selective expression of exogenous opsins by neurons allowing experimental control of their electrical activity using visible light. The interpretation of the results of optogenetic experiments is based on the assumption that light stimulation selectively acts on those neurons expressing the exogenous opsins without perturbing the activity of naive ones. Here, we report that light stimulation, of wavelengths and power in the range of those normally used in optogenetic experiments, consistently reduces the firing activity of naive Mitral Cells (MCs) and Tufted Neurons in the olfactory bulb as well as in Medium Spiny Neurons (MSNs) in the striatum. No such effect was observed for cerebellar Purkinje and hippocampal CA1 neurons. The effects on MC firing appear to be mainly due to a light-induced increase in tissue temperature, between 0.1 and $0.4^{\circ} \mathrm{C}$, associated with the generation of a hyperpolarizing current and a modification of action potential (AP) shape. Therefore, light in the visible range can affect neuronal physiology in a cell-specific manner. Beside the implications for optogenetic studies, our results pave the way to investigating the use of visible light for therapeutic purposes in pathologies associated with neuronal hyperexcitability.
\end{abstract}

\title{
Introduction
}

Since its conception and development at the beginning of this century, optogenetics have rapidly gained prominence in neuroscience research (Williams \& Deisseroth, 2013)(Deisseroth, 2015). This technique uses genetic engineering to selectively express, in specific neuronal populations, light-sensitive proteins (opsins) initially obtained from nonmammalian species (Banghart et al., 2004; Boyden et al., 2005). Exposure of the brain tissue to light at specific wavelengths activates these opsins and gates ionic flux through the cell 
membrane; it then modifies the membrane potential by activating or inhibiting the opsinexpressing neurons. The most widely used exogenous opsins in optogenetic experiments are channelrhodopsin 2 (ChR2) and halorhodopsin ( $\mathrm{NpHR})$. The first is a cation permeable channel that can be activated by visible light in the $350-550 \mathrm{~nm}$ range (experimentally activated mostly around $480 \mathrm{~nm}$, blue light); stimulation of ChR2 produces membrane depolarization and neuronal firing (Gradinaru et al., 2010). The second, NpHR, is a lightactivated ionic pump selective for chloride ions that can be turned on by visible light in the 500-600 nm range (generally activated experimentally around $560 \mathrm{~nm}$, yellow light); stimulation of NpHR produces membrane hyperpolarization and prevents neuronal firing (Gradinaru et al., 2010). Light-controlled firing of neuronal cells using optogenetics is therefore an extremely powerful tool to precisely determine the role of specific neuronal populations in brain physiology, brain pathology and behavior (Fenno et al., 2011; Deisseroth, 2015). The interpretation of optogenetic experiments is based on the assumption that light stimulation selectively acts on those neurons expressing the exogenous opsins; however, several reports have shown that light can, in some cases, affect the physiology of naive neurons. While the action of infrared light (wavelength $>700 \mathrm{~nm}$ ) on neuronal physiology has been known since the early sixties (Arvanitaki \& Chalazonitis, n.d.) and is well documented (Shapiro et al., 2013; Deng et al., 2014), few investigations have examined the effects of light in the visible spectrum on naive brain physiology. Christie et al. (Christie et al., 2013) showed that blue laser light stimulation produces an fMRI signal in a rat cortex; an effect that has been attributed to direct vascular dilatation (Rungta et al., 2017). Moreover, a recent report showed that in mice, laser stimulation at $532 \mathrm{~nm}$ increased the firing activity of prefrontal cortex neurons, in vivo (Stujenske et al., 2015), depending on light power ( $43 \%$ for $10 \mathrm{~mW}, \sim 31 \%$ for $5 \mathrm{~mW}$ and no modification for $1 \mathrm{~mW}$ ). In both cases, the effect of the light appears to be due to an increase in brain tissue temperature. Such light-induced 
temperature increases depend on wavelength, power and duration and could be attenuated by reducing the duty cycle of pulse light stimulation (Stujenske et al., 2015). Finally, our pilot experiments showed that LED stimulation (1 second stimulation with light in 470-570 nm range, with a power of $13 \mathrm{~mW}$ ) consistently reduced the firing activity of Mitral Cells (MCs) recorded in vitro from olfactory bulb (OB) slices (see pilot data at https://osf.io/4fbpt/). Hence, light in the wavelength and power ranges used for optogenetic experiments appears to affect neuronal activity even in the absence of exogenous opsins. Investigating this phenomenon is important for at least two reasons. First, characterization of the action of visible light on naive neurons is crucial for the interpretation of optogenetic experiments, in order to exclude non-specific effects. Second, optogenetics requires gene transfection, limiting its potential therapeutic utilization in humans, for example to prevent or reduce epileptic seizures(Bentley et al., 2013). This limitation would be avoided if the activity of naive neurons could be controlled using light and this would open the way to investigating using visible light for therapeutic purposes.

The goals of the proposed research were the following:

- Reproduce the results reported in the pilot experiment

- Investigate whether and how light-induced inhibition of MC firing is affected by the light's power, wavelength and duration in in vitro conditions.

- Determine the extent of any temperature modifications produced by light stimulation and so get information on potential correlations between this parameter and lightinduced modifications of firing activity.

- Get insights into the cellular mechanisms of light-induced inhibition of MC firing (role of inhibitory network activity, role of G-protein coupled receptors, modification 
of membrane potential $(\mathrm{Vm})$, modification of membrane resistance $\left(\mathrm{R}_{\mathrm{m}}\right)$ ), under in vitro conditions.

- Determine the effects of visible light on the firing activity of neuronal types other than MCs (hippocampal CA1 pyramidal neurons, cerebellar Purkinje neurons and striatal medium spiny neurons) under in vitro conditions.

\section{Methods}

The experiments were performed in three different laboratories, each working on a different preparation; namely: C. Beurrier's IC2N team (IBDM URM7288, Marseille) working on striatal medium spiny neurons (Striatum); M. Canepari's MOTIV team (Laboratoire Interdisciplinare de Physique Grenoble) studying cerebellar Purkinje neurons (Cerebellum) and N. Kuczewski's NEUROPOP team (Lyon Neuroscience Research Center, Lyon) using MCs and tufted cells from the olfactory bulb (OB) as well as hippocampal CA1 pyramidal neurons (Hippocampus). One of the principal purposes of this project was to determine whether the effect observed in the preliminary experiments of light on firing activity, is a generalizable phenomenon and independent of specific experimental conditions; for this reason, each laboratory worked using its own protocol for slice preparation and cell recording. The protocols for electrical and optical stimulations (light wavelength and power) of the neurons were the same in all laboratories.

The team FORGETTING (Lyon Neuroscience Research Center, Lyon) that was supposed to carry out the experiment in the hippocampus retired from the project after the stage 1 in principle acceptance. The proposed experiments in the hippocampus were therefore performed by N. Kuczewski and M Canepari. 
The detailed experimental procedure (registered protocol), approved in a stage I submission, is available at https://osf.io/sf3eb/.

\section{Animals}

Forty-one male C57B16/J mice (Charles River Laboratories, France) aged between 30 and 90 days were used. Mice were housed in groups of five in standard laboratory cages and kept on a $12 \mathrm{~h} \mathrm{light/dark} \mathrm{cycle} \mathrm{(at} \mathrm{a} \mathrm{constant} \mathrm{temperature} \mathrm{of} 22^{\circ} \mathrm{C}$ ) with food and water ad libitum. Experimental procedures were in accordance with the European Community Council Directive of 22nd September $2010(2010 / 63 / \mathrm{UE})$ on the care, welfare and treatment of animals. Procedures were reviewed by the ethics committee affiliated to the animal facility of the Grenoble university (D3842110001) and Lyon1 university (C2EA).

\section{Electrophysiology.}

\section{Olfactory bulb}

Animals were anesthetized with an intra-peritoneal injection of ketamine $(50 \mathrm{mg} / \mathrm{ml})$ and decapitated. The head was immediately immersed in ice-cold $\left(2-4^{\circ} \mathrm{C}\right)$ carbogenized artificial cerebrospinal fluid (cACSF; composition: $125 \mathrm{mM} \mathrm{NaCl,} 4 \mathrm{mM} \mathrm{KCl,} 25 \mathrm{mM} \mathrm{NaHCO} 3,0.5$ $\mathrm{mM} \mathrm{CaCl} 2,1.25 \mathrm{mM} \mathrm{NaH2PO} 4,7 \mathrm{mM} \mathrm{MgCl} 2$ and $5.5 \mathrm{mM}$ glucose; $\mathrm{pH}=7.4$ ) oxygenated with $95 \%$ O $2 / 5 \% \mathrm{CO} 2$. The osmolarity was adjusted to $320 \mathrm{mOsm}$ with sucrose. The OB was sliced horizontally (400 $\mu \mathrm{m}$ thick) with a vibratome. Slices were incubated in a recovery chamber, at $30 \pm 1{ }^{\circ} \mathrm{C}$ in the dark, using an ACSF solution with a composition similar to the cACSF, except that the $\mathrm{CaCl} 2$ and $\mathrm{MgCl} 2$ concentrations were $2 \mathrm{mM}$ and $1 \mathrm{mM}$, respectively. Slices were transferred to a recording chamber mounted on an upright microscope and continuously perfused with oxygenated ACSF $(4 \mathrm{ml} / \mathrm{min})$ at $30 \pm 1^{\circ} \mathrm{C}$. Neurons were visualized using a microscope (Zeiss axioscope) with a 40x objective (Zeiss Plan- 
APOCHROMAT). Data were acquired with an RK 400 BioLogic amplifier at a sampling frequency of $20 \mathrm{kHz}$ using a 12-bit A/D-D/A converter (Digidata 1440A, Axon Instruments) and PClamp10Axon Instruments acquisition software. Patch-clamp whole-cell recordings were done using borosilicate pipettes with a resistance of 4-8 M $\Omega$. The recording pipette was filled with the following intracellular solution (in $\mathrm{mM}$ ): $126 \mathrm{~K}$-gluconate, $5 \mathrm{KCl}, 10 \mathrm{HEPES}$, 1 EGTA, $1 \mathrm{MgCl} 2,2$ ATP-Na2, 0.3 GTP-Na3, and 10 phosphocreatine; $\mathrm{pH}=7.3,290$ mOsm). The membrane potential, measured with the patch pipette, was corrected for the junction potential $(-15 \mathrm{mV})$. Data analysis was performed using OpenElectrophy (Garcia \& Fourcaud-Trocmé, 2009), SciPy, and MySql database software (open source licenses).

\section{Cerebellum}

The Prefecture of Isere approved the experiments on cerebellar Purkinje neurons, performed at the Laboratoire Interdisciplinaire de Physique (Authorisation n. 38 12 01). Sagittal cerebellar slices $(250 \mu \mathrm{m}$ thick) were prepared from 30-45-day-old C57B16 mice following published procedures (Vogt et al., 2011a, 2011b; Ait Ouares et al., 2016) using a Leica VT1200 (Leica, Wetzlar, Germany). Slices were cut in iced extracellular solution and incubated at $37^{\circ} \mathrm{C}$ for 30 minutes before use. The extracellular solution contained (in $\mathrm{mM}$ ): $125 \mathrm{NaCl}, 26 \mathrm{NaHCO} 3,1 \mathrm{MgSO} 4,3 \mathrm{KCl}, 1 \mathrm{NaH} 2 \mathrm{PO} 4,2 \mathrm{CaCl} 2$ and 20 glucose, saturated with 95\% O2 and 5\% CO2. The intracellullar solution contained (in $\mathrm{mM}$ ): $125 \mathrm{KMeSO} 4,5$ KCl, 8 MgSO4, 5 Na2-ATP, 0.3 Tris-GTP, 12 Tris-Phosphocreatine, 20 HEPES, adjusted to $\mathrm{pH} 7.35$ with $\mathrm{KOH}$. Experiments were performed at $32-34^{\circ} \mathrm{C}$ using an Olympus BX51 microscope equipped with a $60 \mathrm{X} / 1.0$ NA Nikon objective. Patch-clamp recordings were done using a Multiclamp amplifier 700A (Molecular Devices, Sunnyvale, CA). The membrane potential, measured with the patch pipette, was corrected for junction potential $(-11 \mathrm{mV})$ as described by Canepari et al. (Canepari et al., 2010). 


\section{Striatum}

The methodology for striatum slices preparation differ from what proposed in the manuscript approved in stage 1 . This deviation is due to the fact that the new method increase the cell quality and survival in slices prepared from juvenile/adult mice(Ting et al., 2014):

30-45-day-old C57B16 mice were anesthetized with an intra-peritoneal injection of ketamine and xylazine (100 and $10 \mathrm{mg} / \mathrm{kg}$, respectively) and transcardially perfused with an ice-cold $\mathrm{N}$ methyl D-glucamine (NMDG)-based solution containing (in mM): $93 \mathrm{NMDG}, 2.5 \mathrm{KCl}, 1.2$ $\mathrm{NaH}_{2} \mathrm{PO}_{4}, 30 \mathrm{NaHCO}_{3}, 20 \mathrm{HEPES}, 20$ glucose, $10 \mathrm{MgCl}_{2}, 93 \mathrm{HCl}, 2$ Thiourea, 3 sodium pyruvate, $12 \mathrm{~N}$-acetyl cysteine and $0.5 \mathrm{CaCl}_{2}$ (saturated with $95 \% \mathrm{O}_{2}$ and $5 \% \mathrm{CO}_{2}, \mathrm{pH} 7.2$ 7.4). Immediately after decapitation the brain was removed, chilled in ice-cold oxygenated NMDG-based solution before cutting coronal slices $(250 \mu \mathrm{m})$ with a vibratome (Leica, VT1000S) at $4^{\circ} \mathrm{C}$. Slices were immediately transferred to recover in NMDG-based solution at $35^{\circ} \mathrm{C}$ for $5 \mathrm{~min}$ and then stored for at least $1 \mathrm{~h}$ at room temperature in normal ACSF (composition (in mM): $126 \mathrm{NaCl}, 2.5 \mathrm{KCl}, 1.2 \mathrm{MgCl}$, $1.2 \mathrm{NaH} 2 \mathrm{PO} 4,2.4 \mathrm{CaCl} 2,25$ NaHCO3 and 11 glucose), to which $250 \mu \mathrm{M}$ kynurenic acid and $1 \mathrm{mM}$ sodium pyruvate had been added. For the recordings, slices were transferred one at a time to a submersion-type chamber and perfused continuously with warm ACSF $\left(32-34^{\circ} \mathrm{C}\right)$ at a rate of $3 \mathrm{ml} / \mathrm{min}$. All solutions are continuously equilibrated with $95 \% \mathrm{O} 2 / 5 \% \mathrm{CO} 2$. Neurons were visualized using an upright microscope (Nikon Eclipse FN1) equipped with a DIC optic and using a 40x water-immersion objective. Electrophysiological recordings were made with a Multiclamp 700B amplifier and Clampex 10.6 software (Molecular Devices). Patch-clamp electrodes (4-6 $\mathrm{M} \Omega$ ) were filled with an intracellular solution containing (in $\mathrm{mM}$ ): $126 \mathrm{KMeSO} 4,14 \mathrm{KCl}, 3$ 
$\mathrm{MgCl} 2,0.5 \mathrm{CaCl} 2,5$ EGTA, 10 HEPES, $2 \mathrm{NaATP}$ and $0.5 \mathrm{mM} \mathrm{NaGTP,} 10 \mathrm{Na}-$ Phosphocreatine, $\mathrm{pH}$ adjusted to 7.25 with $\mathrm{NaOH}$ and osmolarity adjusted to 270-280 $\mathrm{mOsm} / \mathrm{L}$.

CA1 areas of the hippocampus

The equipment used for slice preparation differs from that presented in the manuscript approved in stage 1 . Such divergence is due by the fact that these experiments were done in a different laboratory of that originally planned.

Coronal brain slices $(350 \mu \mathrm{m})$ were cut from 8-10-week-old C57B16 mice with a vibratome (VT1000S, Leica) and incubated at $33^{\circ} \mathrm{C}$ for $30 \mathrm{~min}$ and then left for at least $30 \mathrm{~min}$ at room temperature in artificial cerebrospinal fluid (aCSF) containing (in $\mathrm{mM}$ ): $124 \mathrm{NaCl}, 10$ glucose, $1.25 \mathrm{NaH} 2 \mathrm{PO} 4,2.5 \mathrm{KCl}, 26 \mathrm{NaHCO} 3,1.3 \mathrm{MgCl} 2$, and $2.5 \mathrm{CaCl} 2$ and superfused with a gaseous mixture $(95 \% \mathrm{O} 2$ and $5 \% \mathrm{CO} 2)$. Slices were transferred to a recording chamber maintained at $33^{\circ} \mathrm{C}$. For whole-cell current-clamp recordings of $\mathrm{CA} 1 / \mathrm{CA} 3$ pyramidal cells, the patch pipettes were made of borosilicate glass $(\sim 5 \mathrm{M} \Omega)$ filled with the following solution (in $\mathrm{mM}$ ): 120 potassium methyl sulphate, 10 Na-phosphocreatine, 0.1 $\mathrm{CaCl} 2,2 \mathrm{MgCl}$, 10 HEPES, 1 EGTA and 2 Na-ATP, $0.5 \mathrm{mM} \mathrm{NaGTP(300} \mathrm{mOsm,} \mathrm{pH} \mathrm{=} \mathrm{7.2).}$ Neurons were visualized using a microscope (Zeiss axioscope) with a 40x objective (Zeiss Plan-APOCHROMAT). The data were acquired with the RK 400 BioLogic amplifier at a sampling frequency of $20 \mathrm{kHz}$ using a 12-bit A/D-D/A converter (Digidata 1440A, Axon Instruments) and PClamp10Axon Instruments acquisition software.

\section{Temperature measurement}

Slice and bath temperature were measured and controlled using a ThermoClamp ${ }^{\mathrm{TM}}-1$ from Atomate Scientific. 


\section{Optical stimulation}

Olfactory bulb, cerebellum and hippocampus

With the olfactory bulb two wavelength ranges were used: 1) 430-495 nm peaked at $470 \mathrm{~nm}$ (blue light) using a Dual Port OptoLED, CAIRN, UK, dichroic mirror 495 nm, Zeiss; 2) 470$570 \mathrm{~nm}$ peaked at $540 \mathrm{~nm}$ (green/yellow light) using a white LED Dual Port OptoLED, CAIRN, UK with excitation $470 \mathrm{~nm}$ high-pass filter and $570 \mathrm{~nm}$ dichroic mirror, Zeiss. Only blue light was used in the cerebellum and hippocampus experiments.

\section{Striatum}

Controlled by the acquisition software, blue light $(470 \mathrm{~nm})$ was delivered from a Spectra Light Engine (Lumencor, Optoprim) and connected to the back aperture of the microscope through a $3 \mathrm{~mm}$ liquid-core fiber, producing a wide-field exposure around the recorded cell. Its power on leaving the microscope objective was measured using a PM100 power meter via a S130C probe (Thorlabs).

\section{Proposed experiments:}

\section{Experimental procedure:}

The present project is divided in two stages:

Stage one is aimed at reproducing the results observed in the pilot experiment, namely a reduction of $\mathrm{MC}$ firing frequency produced by light illumination in 470-570 nm range (green/yellow) during whole-cell recordings (stage 1 manuscript: https://osf.io/sf3eb/, figure 4), and determining if these results depend on light intensity and/or light wavelength. 
Stage 1 experiment 1: to determine whether the effect on MC firing activity produced by light stimulation in 470-570 $\mathrm{nm}$ range (green/yellow) depends on light intensity, we will use three light intensities: 13mW, $5 \mathrm{~mW}$ and $1 \mathrm{~mW}$ (stage 1 manuscript: https://osf.io/sf3eb/, figure 4A). The first is the power used in the pilot experiments; the second and the third that produced an increase or no modification of firing activity in cortical neurons in vivo, respectively (Stujenske et al., 2015). All these light intensities are commonly used in optogenetic experiments.

Stage 1 experiment 2: to determine whether the light effect depends on light wavelength, we will use the same protocol as the one described in experiment 1 but with 430-495 nm light stimulation (blue).

\section{Stage one analysis:}

For each recorded neuron and for each intensity (depicted in figure 4A), the effect of light will be assessed by a statistical comparison between the number of spikes in NO LED and LED conditions, using a paired unilateral test. For each comparison, the results will be reported as shown in figure $2 \mathrm{~B}$. The sample size for this experiment is based on the pilot experiments. As shown in figure $2 \mathrm{D}$, the estimated effect size of light on $\mathrm{MC}$ firing is $\mathrm{ES}=-0.9$. Using this value as target effect size, we have chosen the sweep sample size, i.e. the number of traces acquired for each neuron recorded (see stage 1 manuscript: https://osf.io/sf3eb/ fig 1A, 2A), to be $n=30$. This value predicted a statistical power of 0.999 . It should be noted that even if the real ES is closer to the lower limit of the $95 \% \mathrm{CI}(\mathrm{ES}=-0.6)$, the statistical power is still above $0.9(\mathrm{ES}=0.94)$. The authenticity of the effect will also be assessed by assuming that the null hypothesis, i.e. "light has no effect", is true and by computing the probability to have observed by chance at least $\mathrm{n}$ statistically significant results over $\mathrm{N}$ total comparisons. This probability value is obtained by the following equation:

Equation 1: $P(h \geq n)=\sum_{h=n}^{N} C(N, h) p^{h}(1-p)^{N-h}$ 
Where $\mathbf{p}=0.05, \mathbf{N}=$ the total number of recorded neurons and $\mathbf{n}=$ the number of neurons for which the $\mathrm{p}$-value is $<0.05$. For our pilot experiment, where $\mathrm{N}=11$ and $\mathrm{n}=7$ (see stage 1 manuscript: https://osf.io/sf3eb/,figure $2 \mathrm{~B}$ ), this probability is equal to $2 * 10^{-7}$. In other words, the probability that the observed significant results are due exclusively to a repetition of type I error is $2 * 10^{-7}$. Three populations analyses, one for each intensity, will be made by comparing the average number of spikes of each recorded neuron in the NO LED condition to the average number of spikes in the LED condition. The result will be presented as $\%$ of modification, i.e. (average spike LED-average spike NO LED)/average spike NO LED, such as in figure $2 \mathrm{E}$ ).

The size effect of the population analysis showed in figure $2 \mathrm{E}$ is $\mathrm{ES}=-1.07$. Using this estimation as a target ES, a sample size of 20 neurons gives a predicted statistical power of 0.998 .

It should be noted that we expect to reduce the effects of light on MC firing by decreasing light power and by increasing light wavelength $[10,11]$; what would probably reduce the ES when using a light power lower than $13 \mathrm{~mW}$ (the one used in the pilot experiments). For $\mathrm{n}=30$ sweeps in the neuron by neuron analysis and $n=20$ neurons in the population analysis, the statistical power becomes lower than 0.9 for $\mathrm{ES}<0.54$ and $\mathrm{ES}<0.68$, respectively. However, the utilization of equation 1 will still allow to reliably detect genuine light effects even for much lower effects size. For example, with an $\mathrm{ES}=0.2$ the statistical power on neuron by neuron analysis is 0.28 . By repeating the experiment 20 times $(20$ recorded neurons) we should expect to observe $\sim 5$ neurons over 20 for which the p-value $<0.05$. Replacing these values in equation 1 give a probability of 0.002 . Since this is the probability that the observed significant effects are due exclusively to a repetition of type I error, such value will constitute an argument to reject the null hypothesis. 
Stage two will be performed only if stage one will reproduce the results of the pilot experiment. This stage is aimed to investigate: a) the physical and physiological mechanisms of light effects on neuronal firing. b) Whether light effect on $\mathrm{MC}$ firing is a phenomenon generalizable to other neuronal types. For stage two, light wavelength and power will be those that produced the maximum effect in stage 1 experiments. The sample size will be determined upon knowledge of stage 1 results as follows: the neuron analysis and population analysis ES's, observed in the experimental condition (light power and light wavelength) that produce the maximal effect in stage one, will be used to determine the number of sweeps and the number of neurons that give a statistical power equal to 0.95 .

Experiment 4 bis, 6,7 and 8 should be considered exploratory. No statistical power is estimated a priori.

Stage 2 experiment 1-2: The goal is to determine whether the light-induced modification of $\mathrm{MC}$ firing is due to temperature changes.

Stage 2 Experiment 1: Determine the slice temperature modification produced by LED stimulus. The experiment will be performed by putting a temperature probe in the slice. The protocol is depicted in figure 4 B of stage 1 manuscript: https://osf.io/sf3eb/. Statistical effect will be assessed by paired unilateral test between the average slice temperature measured for one second $\left(\mathrm{T}_{\mathrm{Ctr}}\right)$ before light stimulation and the average slice temperature measured during one second of light stimulation $\left(\mathrm{T}_{\mathrm{LED}}\right)$. No electrophysiological recording will be made. For this experiments the two wavelengths of stage 1 will be used. This will allow to correlate an eventual difference between blue and yellow light effects, observed in stage 1 , with an eventual difference in their hitting effects.

Stage 2 Experiment 2: Determine the impact of the slice temperature modification produced by LED stimulus (measured in experiment 1) on MC firing. The protocol is depicted in figure 4 C of stage 1 manuscript: https://osf.io/sf3eb/. The bath temperature in the recording 
chamber will be modified while performing CC recording on MC. Statistical effect will be assessed by umpired bilateral test between the number of action potentials elicited in $\mathrm{T}_{\mathrm{Ctr} 1}$ and the number of action potentials elicited in the condition $T_{L E D}$ spike. The number of action potentials elicited in the condition $\mathrm{T}_{\mathrm{Ctr} 2}$ will be evaluated to assess the reversibility of an eventual effect produced by the temperature modification. No LED stimulation will be made. $\mathrm{MC}$ will be recorded in the same dark ambient light condition used for the other experiments.

Stage 2 experiment 3: The goal is to determine whether the effect of light on MC firing depends on light duration. The protocol used is depicted in figure 4D. The number of spikes in LED1, LED2 and LED3 condition will be compared using ANOVA (repeated measures) followed by post hoc paired test. Paired test comparisons between NO LED and LED1 conditions will be performed in order to make sure of the presence of the light effect. Unilateral test will be used.

Stage 2 experiment 3 bis: The goal is to determine the modification of slice temperature produced by increasing light duration. These results will be correlated to the eventual differences on MC firing activity produced by the modification of light duration (Stage 2 experiment 3). The protocol is similar to that described in Stage 2 Experiment 1 and figure 4B of stage 1 manuscript: https://osf.io/sf3eb/ with $\mathrm{T}_{\text {LED }}$ measured in the last second of light stimulation.

Stage 2 experiment 4: The goal is to determine whether the effect of light on MC firing is a consequence of light-induced increase of inhibitory interneurons activity. The same protocol used in the pilot experiments ( used but with LED stimulation at the power and wavelength that produced the maximal effect in stage 1 experiments and in the presence, in the extracellular solution, of the GABAA receptors antagonist SR-95531 (Gabazine, $5 \mu \mathrm{M}$ ) and of the GABAB receptors antagonist CGP $55845(10 \mu \mathrm{M})$. For each recorded neuron, light effect will be assessed by a statistical 
comparison between the number of spikes in the NO LED and LED conditions, using a paired unilateral test. Population analysis will be performed as previously described.

Stage 2 experiment 4 bis : This experiment will be performed only if, in Stage 2 experiment 4, Gabazine will prevent the light-induced reduction of MC firing. The same protocol described in stage 2 experiment 4 will be used but while recording from Granuler Cells (GC). For each recorded neuron, light effect will be assessed by a statistical comparison between the number of spikes in the NO LED and LED conditions, using a paired bilateral test. Population analysis will be performed as previously described.

Stage 2 experiment 5: The goal is to determine whether the effect of light on MC or GC firing is mediated by the activation of G-protein-coupled receptors. For this reason, the same protocol used in the pilot experiments (stage 1 manuscript: https://osf.io/sf3eb/, figure 2A) will be used with LED stimulation at the power and wavelength that produced the maximal effect in stage 1 experiments. However, in order to block G protein activity, we will replace the GTP-Na3 in the intracellular solution with $2 \mathrm{mM}$ of the non-hydrolyzable GDP analog Guanosine 5'-[ $[\beta$-thio]diphosphate trilithium salt (GDP $\beta S)$, a $\mathrm{G}$ protein inhibitor. For each recorded neuron, light effect will be assessed by a statistical comparison between the number of spikes in the NO LED and LED conditions, using a paired unilateral test. The cellular type recorded in this experiment will depend on the outcome of stage 2 experiment 4 . If GC is recorded, the sample size will be determined based on the effect size calculated from stage 2 experiment 4 bis, to give a statistical power equal to 0.95 .

Stage 2 experiment 6: The goal is to determine whether the light modifies neuronal membrane conductance and/or membrane resistance $(\mathrm{Rm})$. The experiments will be performed in voltage-clamp configuration with neurons maintained at $-60 \mathrm{mV}$. The protocol is depicted in figure $4 \mathrm{C}$. The effect of light on holding current will be quantified by comparing the average current in the control period with the average current during the one second light 
stimulation (LED1). Light effect on Rm will be assessed comparing Rm in No LED to Rm in LED2. $\mathrm{Rm}$ is calculated according to Ohm's law $\mathrm{R}=\mathrm{V} / \mathrm{I}$; where $\mathrm{I}$ is median current in the last $100 \mathrm{~ms}$ of hyperpolarizing step and $\mathrm{V}=-5 \mathrm{mV}$. Statistical effect will be assessed by paired bilateral test. The cellular type recorded in this experiment will depend on the outcome of stage 2 experiment 4 . If GC is recorded, the sample size will be determined based on the effect size calculated from stage 2 experiment 4bis, to give a statistical power equal to 0.95 .

Stage 2 experiment 7: The goal is to determine whether the light modifies the membrane potential $(\mathrm{Vm})$ and/or the action potential (AP) parameters. The experiments will be performed in current-clamp configuration at resting membrane potential or after slight hyperpolarization to prevent spontaneous firing. The protocol is depicted in figure 4E. Light effect on $\mathrm{Vm}$ will be assessed by comparing the average $\mathrm{Vm}$ in the control period (1 second before light stimulation) to the average Vm during 1 second of light stimulation (LED1). Statistical effect will be assessed by paired bilateral test. Light effect on AP will be assessed by generating a single action potential by a $5 \mathrm{~ms}$ positive current injection both in the absence (NO LED) and presence (LED) of light. Two AP parameters will be evaluated: AP amplitude, calculated as the difference between AP peak and the average Vm in the $100 \mathrm{~ms}$ that precedes the current step, and AP latency, calculated as the time between the beginning of the current step and AP peak(Duménieu et al., 2015). Statistical effect will be assessed by paired bilateral test. For the experiments on AP, the $\alpha$ risk, corrected for multiple comparison (2), will be $\alpha$ $=0.025$. The cellular type recorded in this experiment will depend on the outcome of stage 2 experiment 4 . If GC will be recorded, the sample size will be determined based on the effect size calculated from stage 2 experiment 4bis, to give a statistical power equal to 0.95 .

Stage 2 experiment 8: Determine the light effect on the firing activity of tufted cells in the OB, hippocampal CA3/CA1 pyramidal neurons, cerebellar purkinje neurons and striatal medium spiny neurons. These experiments will be performed in three different laboratories. 
The same protocol used in the pilot experiments (stage 1 manuscript: https://osf.io/sf3eb/, figure 2A) will be used but with LED stimulation at the power and wavelength that produced the maximal effect in stage 1 experiments. For each recorded neuron, light effect will be assessed by a statistical comparison between the number of spikes in the NO LED and LED conditions, using a paired unilateral test. Population analysis will be performed as previously described. For each of the cellular types investigated the results will be graphically presented as in figure 2. For these experiments, the $\alpha$ risk, corrected for multiple comparison (4), will be $\alpha=0.0125$ and the sample size will be adapted to keep the statistical power $=0.95$.

\section{Statistics:}

Statistical comparisons will be performed using parametric or non-parametric tests depending on the normality of distributions, which will be assessed by D'Agostino Pearson test: a paired t-test or a Wilcoxon signed-rank test will be used for normal and non-normal distributions, respectively. Since our working hypothesis is that light produces a decrease of neuronal firing, as shown by the pilot data, unilateral test will be used to compare the light effects on neuronal firing. Experiments in which no a priori hypothesis is made (light action on Vm, Rm and AP parameters), data will be compared using a bilateral test. For normally distributed data the ES is calculated as Cohen's d effect size (the mean of the sample difference divided by the standard deviation of sample difference). For non-normal data distributions, the effect size $r$ is calculated using Kerby simple difference formula (Kerby, 2014) as follow: $r=(S-t) / S-t / S$, where $\mathrm{t}=$ the test statistic, is the minimal rank sum; $\mathrm{S}$ is the total rank sum and $\mathrm{S}-\mathrm{t}$ is the maximal rank sum. The $95 \% \mathrm{CI}$ for the Choen $\mathrm{d}$ ES is calculated using the ci.sm function of the R MBESS package. Statistical power and sample size are calculated by using G*Power 3.1.9.2 (Faul et al., 2007) (Dusseldorf university). Results are presented using the following notation $\mathbf{a}[\mathbf{b}, \mathbf{c}]$ where $\mathbf{a}$ is the data average and $\mathbf{b}$ and $\mathbf{c}$ the lower and the higher limit of $95 \%$ CI, respectively All data analysis will be graphically represented as shown in stage $\mathbf{1}$ 
manuscript: https://osf.io/sf3eb/, figure 3. Bayesian statistics were performed using Jasp software (JASP Team (2017). JASP (Version 0.8.5)[Computer software])

\section{Exclusion criteria}

Cells were excluded from experiments when i) their resting membrane potential $\left(\mathrm{V}_{\text {rest }}\right)$ was above $-50 \mathrm{mV}$, ii) when the step depolarization in the no-LED condition did not elicit at least 10 action potentials (AP), ii) when the starting Vm (calculated in the $100 \mathrm{~ms}$ preceding the depolarizing current step) in the no-LED condition differed by more than $5 \mathrm{mV}$ from the starting Vm in the LED condition or iv) when the access resistance was higher than $50 \mathrm{M} \Omega$. Cells were a priori excluded from the analysis when the defined sweep sample size was not reached due to a loss or degradation of neuronal whole-cell recording i.e. for experiments performed in the current-clamp configuration a depolarization of $\mathrm{V}_{\text {rest }}$ above $50 \mathrm{mV}$ and for the voltage-clamp condition an access resistance greater than $50 \mathrm{M} \Omega$.

\section{Results}

All electrophysiological raw traces and data analysis are accessible on the Open Science Framework web site (https://osf.io/mrw93/).

\section{Changes in MC firing with light power (Stage 1 experiments 1-2)}

In order to assess whether the light-induced reduction in MC firing activity observed in the preliminary data depends on light wavelength and/or power we applied the protocol depicted in figure 1A for two different stimuli, 430-495 nm light (blue LED) and 470-570 nm light (green/yellow LED) ( $\mathrm{n}=20 \mathrm{MC}$ for each type of stimulus, $\underline{\mathbf{5} \text { animals }) . ~ A s ~ s h o w n ~ i n ~ F i g u r e ~} 1$ (see https://osf.io/ejh2u/, https://osf.io/fpd6y/ for the experimental design and supplemental analysis), the reduction in firing frequency produced by LED-stimulation decreased at lower power intensities for both the wavelengths used (power effect: blue LED $p<0.001, F=33$; Yellow LED $\mathrm{p}<0.001, \mathrm{~F}=17$, ANOVA). This decrease applied both to the percentage of 
neurons affected by the light (Fig 1B) as well as to the average effect of the light on the MC population (Fig 1C). While green/yellow light produced a significant reduction in firing at all powers tested, statistical comparison failed to show a significant reduction in MC firing when blue light was used at $1 \mathrm{~mW}(\mathrm{p}=0.17)$. It should however be noted that under this condition we still observed a significant decrease in firing in $35 \%$ of recorded neurons. Applying equation 1 shows that the probability of such a decrease being observed only by chance is $\mathrm{p}=3 * 10^{-5}$ suggesting that the absence of a significant effect in the population analysis is likely due to type II error consequent to a lower effect size at this light power. Comparison of the effect at different wavelengths did not show any difference between blue and green/yellow lights ( $\mathrm{p}=0.75, \mathrm{~F}=0.1$ ANOVA). The Bayesian statistics for this analysis are shown in table 1 . A decline in MC firing due to dialysis of intracellular components in whole-cell recordings could sometimes be observed in our experiments. To check whether this phenomenon contributed to reduction in MC firing when stimulated with light, we made a paired comparison between LED firing of trace acquisitions $n$ and the no-LED firing of trace acquisitions $\mathrm{n}+1$ (shifted analysis). As shown in the exploratory analysis presented in supplementary figure 1 , the results produced by the shifted analysis do not qualitatively differ from those of a normal analysis, indicating that cell dialysis did not impact the quantification of the effect of light on firing activity.

The increase in temperature produced by light is able to inhibit MC firing (Stage 2 experiments 1-2)

The parameter most likely affected by light is temperature. Temperature changes produced by LED stimulation were measured by approaching a temperature probe to the top of OB slices. As shown in figure 2 (A, B), a rapid and gradual increase in temperature was observed during a $1 \mathrm{~s}$ exposure to light. The mean temperature increased linearly with power for the two wavelengths tested (blue light $\mathrm{p}<0.0001 \underline{\mathbf{n}=\mathbf{5}, \mathbf{1} \text { animal; }}$ yellow light $\mathrm{p}=0.01, \underline{\mathbf{n}=\mathbf{4}, \mathbf{1} \text { animal; }}$ 
repeated measurement ANOVA). By plotting the effect of light on MC firing vs. the effect of light on tissue temperature (Fig 2C), a linear covariation of these two parameters was observed, suggesting that light-induced temperature modification and firing reduction are correlated. To confirm this link between temperature and firing, we tested whether variations in temperature affected MC firing frequency by increasing the bath temperature in the recording chamber while monitoring firing activity induced by current pulses, in the absence of light. The average increase in bath temperature was $0.44 \pm 0.04{ }^{\circ} \mathrm{C}$, i.e. similar to that produced by $1 \mathrm{~s}$ LED stimulation at $13 \mathrm{~mW}$. A gradual decrease in firing frequency was produced by heating the recording solution (Fig 3A, $\underline{\mathbf{n}=14}$ MC, 5 animals). The effect of temperature was long-lasting and partially reversed 5 minutes after restoration of the initial bath temperature (Fig 3A). The decrease in firing was significant in $92 \%$ of the recorded MCs (Fig 3B). On average, the temperature increase modified MC firing by $-16[-6,-26] \%$ (Fig 3C), similar to the decrease in firing activity produced by a $1 \mathrm{~s}$ LED stimulation at $13 \mathrm{~mW}$ (see Fig 1 and Fig 2C). All these data suggest that the effects of light on MC firing are mainly due to light-induced temperature increases. In the following experiments, only blue light at 13 $\mathrm{mW}$ was used and the sample size was determined from the results shown in figure 1. Since the effect size (ES) for the action of light on single MCs is -1 [-1.2-0.8] in cell-by-cell analysis, the number of sweep repetitions required to reach a statistical power $=0.95$ is $n=13$. The ES for population analysis is $-1.07[-1.62-0.51]$ and the number of recorded neurons to attain a statistical power of 0.95 is $n=11$, except for experiments illustrated in figure 12 , where correction for multiple comparisons required $n=16$ neurons to attain a power of 0.95 .

\section{MC firing increases with duration of light stimulus (Stage 2 experiments 3-3bis)}

To determine whether the impact of light stimulation on firing activity depends on its duration, we used the protocol depicted in Fig 4A. Unexpectedly, many neurons showed a hyperpolarization of $\mathrm{V}_{\text {rest }}$ associated with a strong decrease in evoked firing activity recorded 
in the no-LED condition when the procedure was repeated. This reduced firing could not be prevented by bringing $\mathrm{V}_{\text {rest }}$ to its initial value through steady current injection, or by increasing the depolarizing stimulus (for an individual example, see supplementary Figure 2). Both the Vm hyperpolarization and firing reduction persisted until the end of the recording session, suggesting that light can sustainably impact cellular physiology in addition to its acute effect on firing during light stimulation. Such long-lasting effects were not observed with short stimuli (1s, figure 1). These effects prevented us from using the number of repetitions compatibles with the exclusion criteria, i.e. at least 10 action potentials in the no light stimulation condition. Consequently, we reduced the number of repetitions required for a cell to be accepted from $n=13$ to $n=10$, but thereby reducing the statistical power to detect the effect in cell-by-cell analysis to $\sim 0.90$. Applying these new criteria, we observed a significant decrease in evoked APs for all light durations $(1,5$ and 10s) but that was more pronounced at

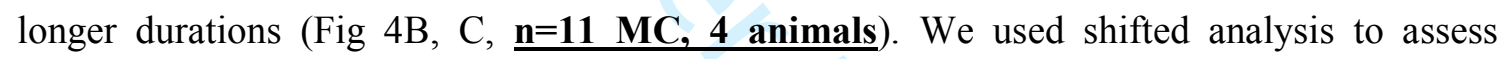
whether the long-lasting effects of light (irreversible Vm hyperpolarization and firing reduction) impact the evaluation of its acute effects (reduction in firing during light pulses) (exploratory analysis, supplementary Figure 3). In this condition, a significant reduction in firing was only observed with 10s light pulses showing that the long-lasting effects mask the acute effects of short pulses. It should however be noticed that, despite the decline in firing, the number of AP during 10-second light stimulation of acquisition trace $n$ is significantly lower than that observed during the 1 -second light stimulation of acquisition trace $n+1$ (supplementary figure 3B), suggesting that acute light effect increased with light duration. We next wondered whether the long-lasting effects of light were also related to temperature changes. Slice temperature increased with increasing light duration $(\mathrm{p}<0.0001$; ANOVA), but such an increase was not significant between 5 and 10s LED stimulations (Fig 5A,B; Bayesian statistics for this analysis are shown in table 2). The maximal light-induced 
temperature increase we observed (around $1{ }^{\circ} \mathrm{C}$ ) is in the range of the physiological fluctuations of the brain (Andersen \& Moser, 1995) or of the bath temperature during in vitro slice experiments. It is therefore very unlikely that the long-term alterations of the physiological properties of MC by lengthy light stimuli are related to temperature modifications.

The effect of light on MC firing does not depend on the inhibitory network and is partially reduced by blocking $G$ protein activity (Stage 2 experiments 4-5)

Temperature can affect MC firing indirectly, for example by increasing the inhibitory synaptic inputs projecting on these neurons. In agreement with this hypothesis, a light-induced increase in olfactory bulb temperature would be responsible for increasing inhibitory interneuron firing that, in turn, would reduce MC activity. This hypothesis is compatible with the differences in temperature sensitivity observed among different neuronal subtypes (Kim \& Connors, 2012). We tested whether the reduction of MC firing when light-stimulated was due to a lightinduced increase of inhibitory activity. This was explored in the presence of $\mathrm{GABA}_{\mathrm{A}}$ and $\mathrm{GABA}_{\mathrm{B}}$ receptors antagonists SR-95531 (Gabazine, $\left.5 \mu \mathrm{M}\right)$ and CGP $55845(10 \mu \mathrm{M})$. Under

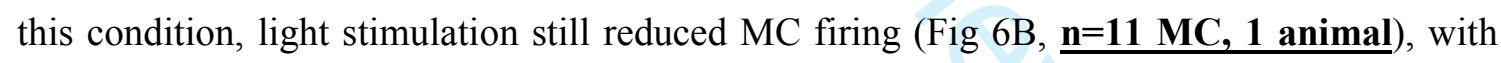
an effect that was comparable to that observed when inhibitory network activity was intact (Fig 6D, Table 3). Hence, the inhibitory network is not involved in the light-induced decrease in MC firing. Extra-retinal photoreceptors expressed in mammal brain neurons (Blackshaw \& Snyder, 1999; Kojima et al., 2011; Nissilä et al., 2012; Fernandes et al., 2013) may participate in this effect on MC firing. Since these neuronal opsins are G-protein-coupled receptors, their possible involvement can be investigated by blocking G-protein signaling, the intracellular target of the photoreceptors. This was achieved by replacing GTP with the nonhydrolyzable GDP analog (GDP $\beta S$ ) in the patch pipette solution. As shown in fig 6C, in this condition, light stimulation still produced a significant reduction of $\mathrm{MC}$ firing frequency 
( $\underline{\mathbf{n}=11}$ MC, 2 animals $)$, although we observed a slight reduction of the effect of light (Ctr vs. GDP $\beta S$ ES $=0.68 ; p=0.032$, Man-Whitney test not corrected for multiple comparisons; fig 6D and table 3 for Bayesian analysis). This result suggests that activation of encephalopsins could contribute to the reduction in MC firing caused by light, but further investigations are required to confirm this observation.

\section{Light stimulation generates an outward current associated with membrane hyperpolarization (Stage 2 experiments 6-7)}

We next investigated the effect of short light stimulation on membrane currents and $\mathrm{V}_{\text {rest. }}$ As shown in figure 7 , LED stimulation $(13 \mathrm{~mW}, 1 \mathrm{~s})$ produced a small $(4.8[1.6,8] \mathrm{pA})$ but consistent outward membrane current in MCs recorded at $-60 \mathrm{mV}$ in the voltage-clamp

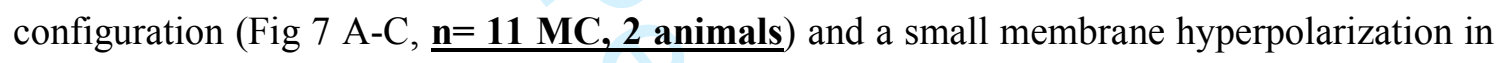

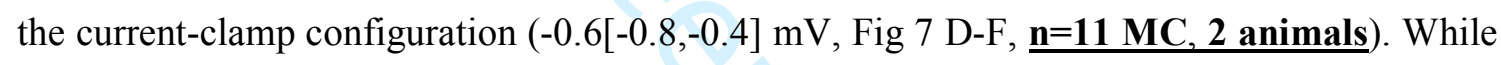
performing these experiments, we noticed a decrease in spontaneous synaptic events during light stimulation in some MCs (Fig 7A, 7D and supplementary fig 4). This synaptic activity is probably excitatory as the reversal potential of GABAergic transmission was $-70 \mathrm{mV}$ in our experimental conditions. One could imagine that the membrane potential modifications observed in the average traces lay behind the suppression of spontaneous excitatory activity by the light but this cannot hold true since the effects on membrane current and voltage were still observed in the presence of the antagonists of synaptic transmission NBQX and APV (unpublished data, but see https://osf.io/pwdjv/,https://osf.io/9k7xj/, and https://osf.io/vrm35/).

The effect of light on membrane resistance $(\mathrm{Rm})$ was evaluated by quantifying the current modification produced by a 1-second hyperpolarizing step of $10 \mathrm{mV}$ (Fig 8A; see https://osf.io/ejh2u/ experiment 6). As illustrated in fig 8B and 8C an apparent increase in Rm was observed during light stimulation. This result can be reconciled with the appearance of 
the outward current depicted in figure 7 only by supposing that the light blocks an unknown steady depolarizing current. Moreover, an increase in $\mathrm{Rm}$ is likely to produce an increase in firing when stimulated, instead of the decrease we observed in MC. We therefore believe that the observed increase is likely due to the fact that our protocol is reliable only in a steady-state condition. Since light stimulation activates an outward current, the latter needs to be subtracted from the current required to produce the voltage step (fig $8 \mathrm{D}$, blue traces) in order to obtain a correct estimation of $\mathrm{R}_{\mathrm{m}}$; this would otherwise be underestimated.

\section{Light stimulation increases AP latency and reduces AP amplitude (Stage 2 experiment 7)}

To determine whether LED stimulation has a direct effect on the cellular mechanisms participating in AP generation, a single action potential was produced by a short $(5 \mathrm{~ms})$ injection of current in the absence of light and at the end of a $1 \mathrm{~s}$ LED stimulation. Analysis of the effect of light on a single AP showed a small reduction in both AP amplitude (-0.6[-0.9,

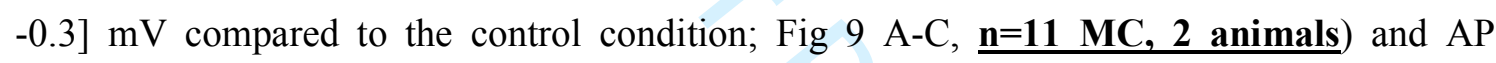
latency calculated from the beginning of current injection $(0.16[0.25,07] \mathrm{ms}$ compared to the control condition; Fig 9 D-F, $\mathbf{n = 1 1}$ MC, 2 animals). Additional exploratory analysis suggested that the AP threshold, rising slope, half-width and afterhyperpolarization (AHP) were also affected by light. Light shifted the AP threshold to more negative membrane potentials and triggered a small increase in the rising slope (Fig $10 \mathrm{~A}$ and B). On the other hand, the half-width of the AP was reduced and the AHP increased (Fig $10 \mathrm{C}$ and D, $\mathbf{n = 1 1}$ $\underline{\text { MC, } 2 \text { animals }}$. As all these parameters mainly depend on sodium and/or potassium channel activation or inactivation (Hodgkin \& Huxley, 1952; Sah \& Faber, 2002; Bean, 2007; Platkiewicz \& Brette, 2010), we can reasonably postulate that light affects AP generation through a modification of $\mathrm{Na}^{+}$and $\mathrm{K}^{+}$channels but further experiments are required to confirm such an interpretation.

\section{Effect of light on firing depends on neuronal types (Stage2 experiment 8)}


Finally, we analyzed whether the firing reduction produced by blue light stimulation on MC was generalizable to other neuronal types using the protocol illustrated in figure $6 \mathrm{~A}$ with tufted cells in the OB, medium spiny neurons in the striatum, cerebellar Purkinje neurons and hippocampal CA1 pyramidal neurons. As shown in figure 11, LED stimulation produced a significant reduction in firing activity in tufted cells $(\underline{\mathbf{n}=\mathbf{1 6}, \mathbf{4} \text { animals }})$ and medium spiny neurons $(\underline{\mathbf{n}=16,8}$ animals $)$ but not in cerebellar Purkinje $(\underline{\mathbf{n}=16,4}$ animals $)$, or CA1 hippocampal neurons ( $\underline{\mathbf{n}=\mathbf{1 6}, \mathbf{3} \text { animals })}$. The possibility that the firing reduction observed in striatal medium spiny neurons was produced by a refractory period was investigated by applying two consecutive depolarizing steps in the absence of LED stimulation. In this condition, no firing reduction was observed $(n=16$, unpublished results). We noticed that light stimulation produced, in some neuronal types, a modification of the AHP that follows the depolarizing step. To quantify the amplitude of the AHP we subtracted the average Vm in the $100 \mathrm{~ms}$ preceding the depolarizing step from the minimum value of $\mathrm{Vm}$ in the $500 \mathrm{~ms}$ that followed the depolarization. Exploratory analysis of this parameter showed a significant light-induced increase in AHP amplitude in tufted cells (no-LED -3.8 [-4.7, -2.7] mV; LED 4.1 $[-5.1,-3.1] \mathrm{mV}, \mathrm{n}=16$ ) and a reduction in hippocampal CA1 pyramidal neurons (no-LED $-4.9[-5.5,-4.3] \mathrm{mV}$; LED -4.6 [-5.3, - 3.9] $\mathrm{mV}, \mathrm{n}=18$ ). No effect was observed in MC (noLED -1.9 [-2.3, -1.5] mV; LED -2.0 [-2.4, -1.6] mV, n=20) or Purkinje neurons (no-LED $1.9[-2.3,-1.5] \mathrm{mV}$; LED -1.7 [-2.0, -1.4] $\mathrm{mV}, \mathrm{n}=18$ ) (Fig 12). Although MSNs do not express AHP in normal conditions a significant negative shift of the membrane potential following the depolarizing step was observed after light stimulation (no-LED 0.17 [0.07, $0.27] \mathrm{mV}$; LED $-0.39[-0.53,-0.26] \mathrm{mV}, \mathrm{n}=17$; Fig 12). These results suggest that light can have opposite actions on membrane properties depending on the neuronal type.

\section{Discussion}


The results presented in this report show that light stimulation, in the wavelength and power ranges commonly used for optogenetic experiments, can modify neuronal firing activity depending on light power and cellular type. They suggest that such effects are mainly due to the increase in tissue temperature and they highlight some of the biophysical mechanisms that might be involved.

\section{Light's action on MC neuronal physiology is mainly due to a temperature modification}

Three lines of evidence suggest that the main factor responsible for the light-induced reduction of MC firing is an increase in temperature of the brain tissue:

1- The reduction of neuronal firing produced by light correlates with the light-induced modification of tissue temperature.

2- The modification of tissue temperature produces a reduction of MC firing similar to that produced by light stimulation.

3- The light-induced modifications of membrane potential and action potential parameters are compatible to those produced by temperature modifications reported in the literature.

We have shown that tissue temperature linearly increases with light power reaching an average value of $0.4^{\circ} \mathrm{C}$ at $13 \mathrm{~mW}$. Such an increase is in the range predicted in the model proposed by Stunjenske et al. for the effect of light in in vitro preparations see (Stujenske et al., 2015) fig 3C. When looking at the effect of light on MC firing, a significant reduction was observed even when using a power of $1 \mathrm{~mW}$, a condition producing a temperature increase less than $0.03^{\circ} \mathrm{C}$. The linear covariation between temperature and firing modifications with light power supports the hypothesis that even at this low light-intensity there is a reduction in firing with increasing temperature, suggesting that neuronal physiology can be affected by extremely small variations of brain temperature. A theoretical model predicts reduced warming with an increase in light wavelength in vivo (Stujenske et al., 
2015); here no significant differences were found between the two wavelengths used. At least three factors could account for this discrepancy: a) the partial overlapping of wavelength bandwidth used in the present study; b) the difference in temperature absorption of in vitro vs. in vivo tissue slices (Yaroslavsky et al., 2002; Johansson, 2010); c) an unspecified/unknown factor not taken into account by the model; see (Senova et al., 2017) fig 4. Brain slice temperatures increased with the duration of light stimulation reaching a steady state after approximately 5 seconds. Such an increase in temperature is associated with a reduction in firing. Interestingly, we found that prolonged light stimulation of MCs produced a persistent membrane hyperpolarization associated with a decline in firing. As pointed out before, it is unlikely that these effects are due to tissue warming $\left(\sim 1^{\circ} \mathrm{C}\right)$ associated with long-lasting LED stimulation. Neither can they be due to prolonged warming (10 seconds), since Vm hyperpolarization and the strong decline in firing were not observed when bath temperatures were increased in the absence of light (see fig 3A). This suggests a direct effect of light on neuronal physiology when prolonged light stimulation is used. Membrane hyperpolarization and declining firing could be due to a slow recovery of physiological activity after light stimulation or to a persistent neuronal alteration, possibly due to phototoxicity. It has been proposed that vestiges of non-visual photoreceptors in the mammalian brain can affect neuronal physiology (Kojima et al., 2011; Fernandes et al., 2013). Our results suggest that activation of encephalopsin could participate in this reduction in $\mathrm{MC}$ firing during visible light stimulation (fig 5). However, the statistical evidence is weak (Bayes factor $=1.14$; $\mathrm{p}=0.032$ ) and more robust results are required to support this evidence. Moreover, we did not observe any light-induced modifications of firing in the Purkinje cells, a neuronal type that strongly expresses encephalopsin (Blackshaw \& Snyder, 1999). Our results cannot exclude the possibility of visible light-activated encephalopsins acting on neuronal parameters other 
than firing activity, as well as the possible role of these opsins when stimulated by ultraviolet light (Kojima et al., 2011).

\section{Biophysical explanation for the effect of light}

The effect of light on membrane properties and action potential parameters were investigated with MCs.

LED-stimulation generated an outward current producing a small but consistent membrane hyperpolarization, an increase in AP latency and a decrease in AP amplitude. Moreover, our exploratory analysis suggests that light stimulation increases the AP rising slope, reduces AP duration and increases the post-spike AHP. Assuming that the observed effects are due to light-induced temperature modifications, it is worth discussing the present results by taking into account the effects produced by temperature variations on neuronal physiology as reported in the literature. A temperature increase has shown to affect neuronal $V_{\text {rest }}$ in different ways, depending on the brain structure and the animal species. In guinea pig hippocampal $\mathrm{CA} 1$ pyramidal neurons held at different temperatures $\left(27^{\circ} \mathrm{C}\right.$ or $\left.37^{\circ} \mathrm{C}\right)$, no significant modifications of $\mathrm{V}_{\text {rest }}$ were observed (Thompson et al., 1985), while in mouse hippocampal neurons, heating from $30^{\circ} \mathrm{C}$ to $41^{\circ} \mathrm{C}$ produced a membrane depolarization between 0.8 and $1.5 \mathrm{mV} /{ }^{\circ} \mathrm{C}$, depending on the neuronal type recorded (Kim \& Connors, 2012). Conversely, a temperature increase from $12^{\circ} \mathrm{C}$ to $31^{\circ} \mathrm{C}$ in a rat visual cortex produced a membrane hyperpolarization of $-1.3 \pm 0.09 \mathrm{mV} /{ }^{\circ} \mathrm{C}$ (Volgushev, Vidyasagar, Chistiakova, \& Eysel, 2000; Volgushev, Vidyasagar, Chistiakova, Yousef, et al., 2000), a result similar to ours with MCs. This result is comparable to the estimated voltage/temperature modification in the present study $\left(-0.6 \mathrm{mV} / 0.34^{\circ} \mathrm{C}=-1.7 \mathrm{mV} /{ }^{\circ} \mathrm{C}\right)$. A temperature increase was also shown to affect AP shape in a similar way to that produced by LED-stimulation of MCs. In particular, a decrease of AP amplitude and duration with increasing temperature was observed in cortical and hippocampal neurons(Thompson et al., 1985; Volgushev, Vidyasagar, 
Chistiakova, Yousef, et al., 2000), as well as an increase in AP latency (Volgushev, Vidyasagar, Chistiakova, Yousef, et al., 2000), rising slope (Thompson et al., 1985) and a decrease in spike threshold (Volgushev, Vidyasagar, Chistiakova, Yousef, et al., 2000). The biophysical mechanisms associated with temperature changes were minutely analyzed by Volgushev et al. who suggest that the modification in $\mathrm{V}_{\text {rest }}$ is mainly due to an increase of the ratio for the permeability of the potassium and sodium leak channels (Volgushev, Vidyasagar, Chistiakova, Yousef, et al., 2000). It should be noted that a temperature dependent increase in the activity of the $\mathrm{Na}^{+}-\mathrm{K}^{+}$pump could also contribute to the observed membrane hyperpolarization(Bates \& Mackillop, 1985). On the other hand the modification of AP shape with changing temperature has been ascribed to an increase in amplitude, duration and steepness of voltage dependent potassium currents and to an increase in amplitude but a decrease in the width of voltage-dependent sodium currents (Volgushev, Vidyasagar, Chistiakova, Yousef, et al., 2000). Similar mechanisms could explain the effect of light on the AP shape in MCs, as well as the observed increase of the fast AHP, mainly due to the activation of voltage-dependent potassium channels in these neurons (Duménieu et al., 2015).

Even though evaluating the effect of light on synaptic transmission was behind the objectives of the present study and requires further investigation, a consistent decrease of putative spontaneous glutamatergic activity was observed in several recorded MCs. A reduction of excitatory synaptic activity could be the consequence of the decrease of spontaneous firing on neighboring MCs and tufted cells as well as the consequence of the reduction of their AP duration and amplitude (Geiger \& Jonas, 2000; Rama et al., 2015). A direct effect of heat or light on neurotransmitter release probability and/or postsynaptic-receptor activation, could also be implicated in the observed effect (Volgushev, Vidyasagar, Chistiakova, \& Eysel, 2000; Leszkiewicz \& Aizenman, 2003). 
The reported effects of light on MC membrane potential, AP proprieties and synaptic activity could all account for the observed reduction of spontaneous and evoked firing activity produced by LED stimulation. Indeed, the outward current so generated would bring the membrane potential away from spike threshold and, although our experimental design did not allow us to evidence this, would probably result in reduced membrane resistance with a consequent reduction in neuronal excitability. This latter hypothesis is in line with the reported reduction of membrane resistance produced by a temperature increase (Thompson et al., 1985; Volgushev, Vidyasagar, Chistiakova, Yousef, et al., 2000). The increase of AP latency and AHP amplitude would also promote a reduction in firing in MCs (Duménieu et $a l ., 2015)$. Finally, the apparent reduction of glutamatergic transmission and in particular the recurrent synaptic excitation, will also lead to a reduction in MC firing (Salin et al., 2001).

We found that LED-stimulation affects both firing activity and AHP amplitude in different and even opposing ways, depending on the neuronal type. Interestingly the light-induced decrease in AHP that we observed in mouse CA1 pyramidal cells is reminiscent of the temperature-induced decrease of the AHP observed in guinea pig CA1 neurons (Thompson et al., 1985). In the latter report the AHP reduction was associated with a decrease in firing adaptation. In the present study we did not evaluate the effect of light on CA1 firing adaptation, focalizing our attention only on the average firing activity, but the raw traces of our experiments are available at https://osf.io/kp34r/. Interesting too the exploratory analysis suggests that in MCs, LED- stimulation increases AHP amplitude following a single AP (Fig 10 D) but not a long depolarizing step (Fig 12). If confirmed these results suggest that light would act differentially on the potassium channels participating in early and medium AHP (Sah \& Faber, 2002). The heterogeneity of the action of light on different neuronal types and parameters is reminiscent of the heterogeneous effect produced by temperature modification reported in the literature and discussed previously and could be due to differences in the 
experimental conditions and/or the biophysical characteristics of the different neuronal types (Kim \& Connors, 2012).

\section{Conclusions}

The results of the present study have three main implications.

The first concerns the ability of optical stimulation, in the range used for optogenetic applications, to affect the neuronal physiology of wild-type neurons. Our results are in agreement with a previous report showing the sensitivity of prefrontal cortex neurons to lightstimulation in vivo (Stujenske et al., 2015). It has been argued that the parameters used in this study (30 s of 5-10 $\mathrm{mW}$ continuous LED-stimulation) that led to a temperature increase of 1-2 ${ }^{\circ} \mathrm{C}$, are not representative of those commonly used in optogenetics experiments (Senova et al., 2017). However, even more canonical stimulation patterns, i.e. pulsed light at a reduced duty cycle, can still produce tissue warming between 0.1 and $0.8^{\circ} \mathrm{C}$ in vivo (Stujenske et al., 2015; Senova et al., 2017). These temperature modifications are sufficiently large to affect the firing activity of some neuronal types recorded in the present study. Thus, controls tests on wildtype animals/neurons should be mandatory to confirm the specificity of the observed effects in optogenetics experiments.

The second implication concerns the sensitivity of some neuronal types to very small variations of tissue temperature, with less than $0.1^{\circ} \mathrm{C}$ sufficient to modify the firing activity of MCs. Since the brain is submitted to daily physiological temperature fluctuations that can reach a few degrees Celsius (Andersen \& Moser, 1995), our results raise the question of whether, and how, the possible modifications in firing activity produced by physiological temperature fluctuations have an impact on cognitive functions.

The third implication concerns a possible therapeutic application of optical stimulation. The potential of optogenetics to treat certain brain pathologies has been documented in animal models (Bentley et al., 2013; Vann \& Xiong, 2016), but its application in humans is limited 
by the need to express exogenous opsins. Our demonstration of the sensitivity to light of naive neurons, not limited by this constraint, suggests that light stimulation alone could be used to inactivate or activate selective brain regions during pathological manifestations. Since blue-light delivered in the ear canal of healthy humans appears to penetrates the skull and modifies the event-related potential recorded with an EEG (Sun et al., 2016), visible light could potentially be used as a non-invasive tool to study human brain activity for therapeutic or research purposes, but further investigations are necessary to better elucidate the action of light at cellular and cognitive levels.

Acknowledgements: This work was supported by the CNRS, Inserm, and Lyon 1 University. We would like to thank: Nicolas Fourcaud-Trocmé and Samuel Garcia for helping produce the scripts for data analysis; Jean-Christophe Comte for helpful suggestions and Emilie Mathian for her contribution to the preliminary experiments.

Competing Interests: the authors declare no competing financial interests.

Author Contributions: K.Ait Ouares performed the experiment with Purkinje neurons. C. Beurrier supervised the experiments with MSNs, analysed the data and co-wrote the article. M. Canepari supervised the Purkinje and CA1 neurons, analysed the data and co-wrote the article. G. Laverne performed the MSN experiments. N. Kuczewski performed the olfactory bulb and CA1 neuron experiments, analysed the data and co-wrote the article.

Data Accessibility: Raw data and analyses are accessible on the Open Science Framework web site (https://osf.io/mrw93/, DOI 10.17605/OSF.IO/MRW93).

Abbreviations: AHP, afterhyperpolarization; AP, action potential; EPSP, excitatory postsynaptic potential; ES, effect size; MCs, Mitral Cells; MSNs, Medium Spiny Neurons; Rm, membrane resistance; Vm, membrane potential; Vrest, resting membrane potential. 


\section{Figure legends}

Figure 1: The reduction of the evoked MC firing by LED stimulation depends on light power but not wavelength. A) Representative example of LED effect on evoked MC firing. B) Cellby-cell analysis depicting the number and the percentage of neurons for which the LED stimulation (blue or yellow light) produce à significant $(\mathrm{p}<0.05)$ reduction in $\mathrm{MC}$ firing, compared to no-LED condition. C) Population analysis of the effect of blue and yellow LED stimulation on MC firing. Horizontal lines represent average. Error bars represent 95\% CI. Gray bars in A depict the time of LED stimulation. $n=20$ MC. ES=Effect Size

Figure 2: Tissue temperature modifications produced by LED stimulation at different powers and wavelengths. A) Example of temperature modifications induced by blue LED stimulation. B) Average temperature modification, compared to pre-light temperature, during one second stimulation with blue and yellow LEDs. p-values refer to comparisons between light and pre-light temperatures. C) Covariation of the effects of light on MC firing (data from figure 1) and slice temperature. Error bars represent $95 \%$ CI. Blue light $n=5$; yellow light $n=4$.

Figure 3: MC firing is reduced by temperature increase. A) Time plot of the relative modification of the number of evoked APs produced by the increase of temperature in the recording chamber. The number of APs was normalized to the average number of APs in the first 600 seconds. Error bars represent sem. Vertical dashed line represents the start time for chamber warming. Right; representative traces. B) Cell-by-cell analysis of temperature effect on MC firing. Empty dots depict MCs for which no significant difference was observed between the two conditions. Error bars represent 95\% CI. C) Population analysis of temperature effect on MC firing. Error bar represents 95\% CI. n=13 MC. ES=Effect Size

Figure 4: The effect of blue light $(13 \mathrm{~mW})$ on $\mathrm{MC}$ firing increases with stimulus duration. A) Example of the effect of blue light stimulations on evoked MC firing; depolarizing current 
steps inducing firing are omitted from the figure. B) The average number of evoked action potentials decreases with increase in light duration. C) Relative modification of firing frequencies produced by light stimulation at different durations; p-values refers to comparisons between no-LED and LED conditions. Error bars represent 95\% CI. n= 11MC.

Figure 5: Modifications to slice temperatures vary with light duration. A) Example of the modification of $\mathrm{OB}$ slice temperature produced by light stimulation (blue; $13 \mathrm{~mW}$ ) of different durations. B) Average temperature modification produced by the different durations $(\mathrm{n}=4)$. Error bars represent $95 \%$ CI.

Figure 6: Activity in the inhibitory network or activation of extra-retinal photoreceptors are not required for light to modify MC firing. A) Representative example of the protocol used. B) Cell-by-cell analysis of the effect produced by LED stimulation on MC firing in the presence of GABA receptor antagonists. C) Cell-by-cell analysis of the effect produced by LED stimulation on $\mathrm{MC}$ firing in the presence of G-protein blockers GDP $\beta S$ in the intracellular solution. D) Population analysis of light-induced firing frequency modifications in the different conditions (control data are the same as figure $1 \mathrm{C}$ ). In B and C black squares depict MCs for which LED condition is significantly different compared to No-LED condition. Empty dots depict MCs for which no significant difference was observed between the two conditions. Error bars represent 95\% CI. n=11 MC. ES=Effect Size

Figure 7: LED stimulation (blue light; $13 \mathrm{~mW}$ ) generates an outward current and hyperpolarizes MCs. A) Example of light-induced outward current in one neuron. Note the reduction of the spontaneous EPSC during stimulation. B) Cell-by-cell analysis of the modification of the holding current by LED stimulation. C) Population analysis on lightinduced current modification. D) Example of light-induced modification of membrane potential in one neuron. Note the reduction of the spontaneous EPSP during stimulation. E) Cell-by-cell analysis of the modification of the membrane potential by LED stimulation. F) 
Population analysis on light-induced modification of membrane potential. Arrows depict the analysis of the neurons represented in A and D. The black squares depict MCs for which LED condition is significantly different compared to No-LED condition. Empty dots depict MCs for which no significant difference is observed between the two conditions. Error bars represent 95\% CI. n=11 MC. ES=Effect Size

Figure 8: Light stimulation produces an apparent increase in membrane resistance in MC. A) Example of the inward currents generated by a hyperpolarizing voltage step of $10 \mathrm{mV}$ (not shown) in the presence and absence of LED stimulation (blue light; $13 \mathrm{~mW}$ ). B) Cell by cell analysis of the modification of Rm by LED stimulation. The Black squares depict MCs for which LED condition is significantly different compared to No-LED condition. Empty dots depict MCs for which no significant difference is observed between the two conditions. C) Population analysis on light induced Rm modification. Arrows depict analysis of the neuron represented in A. Error bars represent 95\% CI. n=11 MC. ES=Effect Size

Figure 9: LED stimulation (blue light; $13 \mathrm{~mW}$ ) modify AP amplitude and latency. A) Example of effect of light on AP amplitude. The traces are aligned on action potential threshold. B) Cell-by-cell analysis of the modification of AP amplitude by LED stimulation. C) Population analysis on light-induced amplitude modification. D) Example of effect of light on AP latency. The traces are aligned vertically at the membrane potential preceding current injection. E) Cell-by-cell analysis of the modification of AP latency by LED stimulation. F) Population analysis on light-induced latency modification. In B and E the black squares depict MCs for which LED condition is significantly different compared to no-LED condition. Empty dots depict MCs for which no significant difference is observed between the two conditions. Arrows depict analysis of the neurons represented in A and D. Error bars represent $95 \% \mathrm{CI}$. The $\mathrm{p}$ values are corrected for multiple comparisons (2). $\mathrm{n}=12 \mathrm{MC}$. The $\mathrm{p}$ values are corrected for multiple comparisons (2). ES=Effect Size 
Figure 10: Exploratory analysis of LED stimulation (blue light; $13 \mathrm{~mW}$ ) on AP parameters. A) A tendency towards a decrease in AP threshold was produced by light stimulation. B) LED stimulation increases the rising slope of AP. C) Half-width duration of AP is reduced by LED stimulation D) LED stimulation produce an increase of the AHP amplitude that follows one AP; right example of the AHP in light and no-light condition. $n=12$ MC. The p-values are not corrected for multiple comparisons. ES=Effect Size

Figure 11: The effect of light on neuronal firing is dependent on neuronal type. The effect of LED stimulation (blue light; 1s starting from the beginning of the depolarizing step) on evoked firing activity is reported. $\mathrm{MC}=$ Mitral Cells, $\mathrm{MS}=$ medium spiny neurons. Empty dots represent effect of light on neurons for which no statistically significant modifications were observed, black dots represent effect of light on neurons for which statistically significant modifications were observed. MC data are the same as fig $1 \mathrm{C}(\mathrm{n}=20)$. For tufted, MS, Purkinje and CA1 $\mathrm{n}=16$ and the $\mathrm{p}$ values are corrected for multiple comparisons (4). $\mathrm{ES}=$ Effect Size

Figure 12: Exploratory analysis on effect of light on AHP amplitude. A) The effect of LED stimulation (blue light; 1s starting from the beginning of the depolarizing step) on the AHP amplitude observed at the end of the depolarization is reported. Empty dots represent effect of light on neurons for which no statistically significant modifications were observed, black dots represent effect of light on neurons for which statistically significant modifications were observed $\mathrm{MC}=$ Mitral Cells, $\mathrm{MS}=$ medium spiny neurons. Empty dots represent effect of light on single neurons. B) Representative trace of observed AHP modification; blue traces under LED stimulation. $\mathrm{MC} \mathrm{n}=20$; Tufted cells $\mathrm{n}=16$, MSNs $\mathrm{n}=17$, Purkinje neurons $\mathrm{n}=18$ and CA1 neurons $n=18$. The $p$ values are not corrected for multiple comparisons. ES=Effect Size

Supplementary figure 1: The reduction in firing observed during light stimulation is not due to firing rundown; A) Preregistered analysis. left; analysis procedure leading to the results 
presented in figure 1C. Right; analysis of the blue LED stimulation (different representation of the results illustrated in fig 1C ). B) Exploratory analysis. Shifted analysis of the same data set. Left; analysis procedure. The data were analyzed by comparing the modification of firing frequency between the LED conditions of acquisition trace $\mathrm{n}$ and no-LED condition of acquisition trace $\mathrm{n}+1$. Right; shifted analysis results. No qualitative difference is observed between the two types of analysis.

Supplementary figure 2 : The protocol used to assess whether the effect of light on MC firing depends on light duration (shown in figure 4A) producing a membrane hyperpolarization and a rundown of evoked firing activity recorded in no-LED condition. A) Typical evolution of Vrest and the number of evoked action potentials in no-LED condition. Thin arrows depict the repetitions where initial Vrest ( -60 mV) was reset by steady positive current injection. Thick arrow depicts the repetition where the current step evoking the firing was increased in an attempt to recover the initial firing activity. Dashed line depicts the limit marking the minimal number of spikes required to include the repetition in the analysis. B) Three electrophysiological traces for no-LED condition. Up voltage membrane potential; bottom injected step current; number is the repetition. This neuron did not pass the criteria to be included in the analysis (13 repetitions having at least 10 action potentials).

Supplementary figure 3: Firing rundown prevents the correct evaluation of the effects produced by long-lasting LED stimulation. A) Left, example of shifted analysis. The data used for figure 4 were reanalyzed by comparing the modification of firing frequency between the LED conditions of acquisition trace $n$ and no-LED condition of acquisition trace $n+1$. Right, comparison of the results to those of figure 4 show that the rundown affects the capacity to evaluate the acute effect of light on neuronal firing. B) Left, shifted analysis was used to for paired comparisons between LED conditions. Right, despite rundown the firing 
frequency during 10-second LED stimulation of sweeps $\mathrm{n}$ is significantly lower than the firing frequency during 1-second LED stimulation of sweeps n+1. Error bars represent 95\% CI.

Supplementary figure 4: Effect of LED stimulation (13 mW; blue light) on: A) Holding membrane current for all recorded MCs (13-20 traces for each neuron). B) Membrane potential for MCs presenting spontaneous EPSP (13-20 traces for each neuron). Note the apparent decrease of spontaneous excitatory synaptic currents in several of the recorded MCs.

\section{References}

Ait Ouares, K., Jaafari, N., \& Canepari, M. (2016) A generalised method to estimate the kinetics of fast $\mathrm{Ca}(2+)$ currents from $\mathrm{Ca}(2+)$ imaging experiments. J. Neurosci. Methods, 268, 66-77.

Andersen, P. \& Moser, E.I. (1995) Brain temperature and hippocampal function. Hippocampus, 5, 491-498.

Arvanitaki, A. \& Chalazonitis, N. (n.d.) Excitatory and Inhibitory Processes Initiated by Light and Infrared Radiations in Single Identifiable Nerve Cells (Giant Ganglion Cells of Aplysia). .

Banghart, M., Borges, K., Isacoff, E., Trauner, D., \& Kramer, R.H. (2004) Light-activated ion channels for remote control of neuronal firing. Nat. Neurosci., 7, 1381-1386.

Bates, D.A. \& Mackillop, W.J. (1985) The effect of hyperthermia on the sodium-potassium pump in Chinese hamster ovary cells. Radiat. Res., 103, 441-451.

Bean, B.P. (2007) The action potential in mammalian central neurons. Nat. Rev. Neurosci., 8, 451465.

Bentley, J.N., Chestek, C., Stacey, W.C., \& Patil, P.G. (2013) Optogenetics in epilepsy. Neurosurg. Focus, 34, E4.

Blackshaw, S. \& Snyder, S.H. (1999) Encephalopsin: a novel mammalian extraretinal opsin discretely localized in the brain. J. Neurosci. Off. J. Soc. Neurosci., 19, 3681-3690.

Boyden, E.S., Zhang, F., Bamberg, E., Nagel, G., \& Deisseroth, K. (2005) Millisecond-timescale, genetically targeted optical control of neural activity. Nat. Neurosci., 8, 1263-1268.

Canepari, M., Willadt, S., Zecevic, D., \& Vogt, K.E. (2010) Imaging inhibitory synaptic potentials using voltage sensitive dyes. Biophys. J., 98, 2032-2040.

Christie, I.N., Wells, J.A., Southern, P., Marina, N., Kasparov, S., Gourine, A.V., \& Lythgoe, M.F. (2013) $f M R I$ response to blue light delivery in the naïve brain: implications for combined optogenetic fMRI studies. Neurolmage, 66, 634-641.

Deisseroth, K. (2015) Optogenetics: 10 years of microbial opsins in neuroscience. Nat. Neurosci., 18, $1213-1225$.

Deng, W., Goldys, E.M., Farnham, M.M.J., \& Pilowsky, P.M. (2014) Optogenetics, the intersection between physics and neuroscience: light stimulation of neurons in physiological conditions. Am. J. Physiol. Regul. Integr. Comp. Physiol., 307, R1292-R1302.

Duménieu, M., Fourcaud-Trocmé, N., Garcia, S., \& Kuczewski, N. (2015) Afterhyperpolarization (AHP) regulates the frequency and timing of action potentials in the mitral cells of the olfactory bulb: role of olfactory experience. Physiol. Rep., 3. 
Faul, F., Erdfelder, E., Lang, A.-G., \& Buchner, A. (2007) G*Power 3: a flexible statistical power analysis program for the social, behavioral, and biomedical sciences. Behav. Res. Methods, 39, 175-191.

Fenno, L., Yizhar, O., \& Deisseroth, K. (2011) The development and application of optogenetics. Annu. Rev. Neurosci., 34, 389-412.

Fernandes, A.M., Fero, K., Driever, W., \& Burgess, H.A. (2013) Enlightening the brain: linking deep brain photoreception with behavior and physiology. BioEssays News Rev. Mol. Cell. Dev. Biol., 35, 775-779.

Garcia, S. \& Fourcaud-Trocmé, N. (2009) OpenElectrophy: An Electrophysiological Data- and AnalysisSharing Framework. Front. Neuroinformatics, 3, 14.

Geiger, J.R. \& Jonas, P. (2000) Dynamic control of presynaptic Ca(2+) inflow by fast-inactivating $\mathrm{K}(+)$ channels in hippocampal mossy fiber boutons. Neuron, 28, 927-939.

Gradinaru, V., Zhang, F., Ramakrishnan, C., Mattis, J., Prakash, R., Diester, I., Goshen, I., Thompson, K.R., \& Deisseroth, K. (2010) Molecular and cellular approaches for diversifying and extending optogenetics. Cell, 141, 154-165.

Hodgkin, A.L. \& Huxley, A.F. (1952) A quantitative description of membrane current and its application to conduction and excitation in nerve. J. Physiol., 117, 500-544.

Johansson, J.D. (2010) Spectroscopic method for determination of the absorption coefficient in brain tissue. J. Biomed. Opt., 15, 057005.

Kerby, D.S. (2014) The Simple Difference Formula: An Approach to Teaching Nonparametric Correlation. Compr. Psychol., 3, 11.IT.3.1.

Kim, J.A. \& Connors, B.W. (2012) High temperatures alter physiological properties of pyramidal cells and inhibitory interneurons in hippocampus. Front. Cell. Neurosci., 6, 27.

Kojima, D., Mori, S., Torii, M., Wada, A., Morishita, R., \& Fukada, Y. (2011) UV-sensitive photoreceptor protein OPN5 in humans and mice. PloS One, 6, e26388.

Leszkiewicz, D.N. \& Aizenman, E. (2003) Reversible modulation of GABA(A) receptor-mediated currents by light is dependent on the redox state of the receptor. Eur. J. Neurosci., 17, 20772083.

Nissilä, J., Mänttäri, S., Särkioja, T., Tuominen, H., Takala, T., Timonen, M., \& Saarela, S. (2012) Encephalopsin (OPN3) protein abundance in the adult mouse brain. J. Comp. Physiol. A Neuroethol. Sens. Neural. Behav. Physiol., 198, 833-839.

Platkiewicz, J. \& Brette, R. (2010) A threshold equation for action potential initiation. PLoS Comput. Biol., 6, e1000850.

Rama, S., Zbili, M., Bialowas, A., Fronzaroli-Molinieres, L., Ankri, N., Carlier, E., Marra, V., \& Debanne, D. (2015) Presynaptic hyperpolarization induces a fast analogue modulation of spike-evoked transmission mediated by axonal sodium channels. Nat. Commun., 6, 10163.

Rungta, R.L., Osmanski, B.-F., Boido, D., Tanter, M., \& Charpak, S. (2017) Light controls cerebral blood flow in naive animals. Nat. Commun., 8, 14191.

Sah, P. \& Faber, E.S.L. (2002) Channels underlying neuronal calcium-activated potassium currents. Prog. Neurobiol., 66, 345-353.

Salin, P.A., Lledo, P.M., Vincent, J.D., \& Charpak, S. (2001) Dendritic glutamate autoreceptors modulate signal processing in rat mitral cells. J. Neurophysiol., 85, 1275-1282.

Senova, S., Scisniak, I., Chiang, C.-C., Doignon, I., Palfi, S., Chaillet, A., Martin, C., \& Pain, F. (2017) Experimental assessment of the safety and potential efficacy of high irradiance photostimulation of brain tissues. Sci. Rep., 7, 43997.

Shapiro, M.G., Priest, M.F., Siegel, P.H., \& Bezanilla, F. (2013) Thermal mechanisms of millimeter wave stimulation of excitable cells. Biophys. J., 104, 2622-2628.

Stujenske, J.M., Spellman, T., \& Gordon, J.A. (2015) Modeling the Spatiotemporal Dynamics of Light and Heat Propagation for In Vivo Optogenetics. Cell Rep., 12, 525-534.

Sun, L., Peräkylä, J., Kovalainen, A., Ogawa, K.H., Karhunen, P.J., \& Hartikainen, K.M. (2016) Human Brain Reacts to Transcranial Extraocular Light. PloS One, 11, e0149525. 
Thompson, S.M., Masukawa, L.M., \& Prince, D.A. (1985) Temperature dependence of intrinsic membrane properties and synaptic potentials in hippocampal CA1 neurons in vitro. $J$. Neurosci. Off. J. Soc. Neurosci., 5, 817-824.

Ting, J.T., Daigle, T.L., Chen, Q., \& Feng, G. (2014) Acute brain slice methods for adult and aging animals: application of targeted patch clamp analysis and optogenetics. Methods Mol. Biol. Clifton NJ, 1183, 221-242.

Vann, K.T. \& Xiong, Z.-G. (2016) Optogenetics for neurodegenerative diseases. Int. J. Physiol. Pathophysiol. Pharmacol., 8, 1-8.

Vogt, K.E., Gerharz, S., Graham, J., \& Canepari, M. (2011a) High-resolution simultaneous voltage and Ca2+ imaging. J. Physiol., 589, 489-494.

Vogt, K.E., Gerharz, S., Graham, J., \& Canepari, M. (2011b) Combining membrane potential imaging with L-glutamate or GABA photorelease. PloS One, 6, e24911.

Volgushev, M., Vidyasagar, T.R., Chistiakova, M., \& Eysel, U.T. (2000) Synaptic transmission in the neocortex during reversible cooling. Neuroscience, 98, 9-22.

Volgushev, M., Vidyasagar, T.R., Chistiakova, M., Yousef, T., \& Eysel, U.T. (2000) Membrane properties and spike generation in rat visual cortical cells during reversible cooling. J. Physiol., 522 Pt 1, 59-76.

Williams, S.C.P. \& Deisseroth, K. (2013) Optogenetics. Proc. Natl. Acad. Sci. U. S. A., 110, 16287.

Yaroslavsky, A.N., Schulze, P.C., Yaroslavsky, I.V., Schober, R., Ulrich, F., \& Schwarzmaier, H.J. (2002) Optical properties of selected native and coagulated human brain tissues in vitro in the visible and near infrared spectral range. Phys. Med. Biol., 47, 2059-2073. 


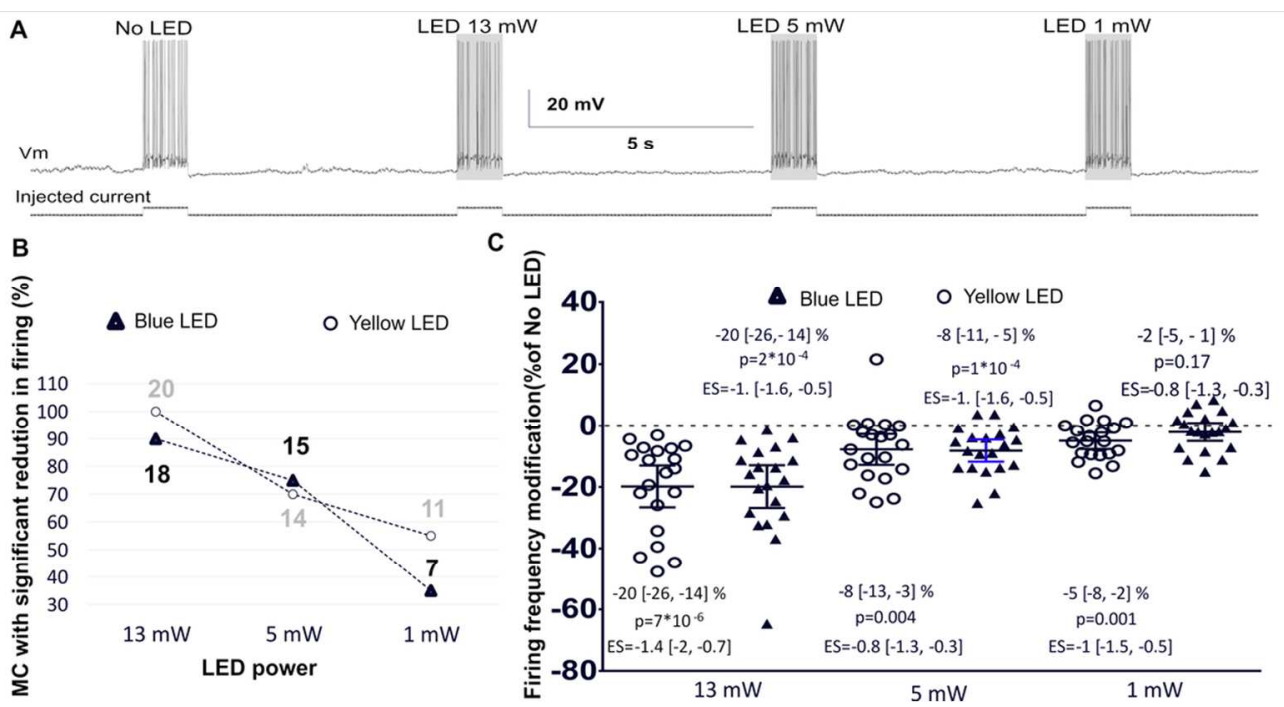

Figure 1: The reduction of the evoked MC firing by LED stimulation depends on light power but not wavelength. A) Representative example of LED effect on evoked MC firing. B) Cell- by-cell analysis depicting the number and the percentage of neurons for which the LED stimulation (blue or yellow light) produce à significant $(\mathrm{p}<0.05)$ reduction in $\mathrm{MC}$ firing, compared to no-LED condition. $\mathrm{C})$ Population analysis of the effect of blue and yellow LED stimulation on MC firing. Horizontal lines represent average. Error bars represent $95 \%$ CI. Gray bars in A depict the time of LED stimulation. $n=20$ MC. ES=Effect Size 


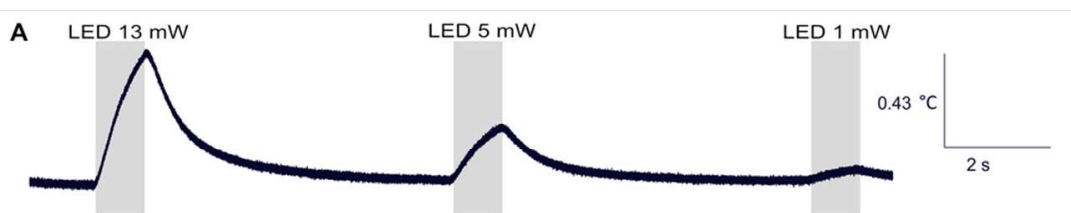

B

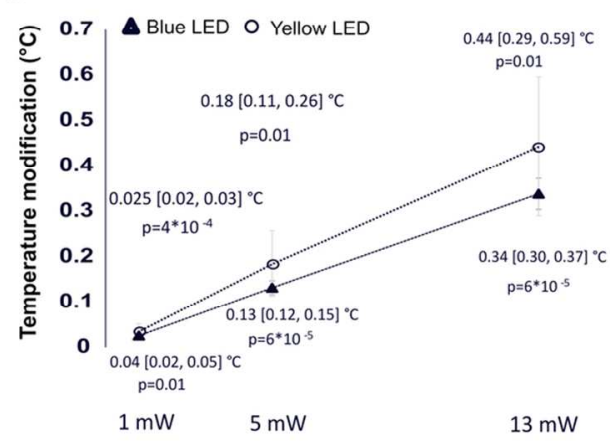

c

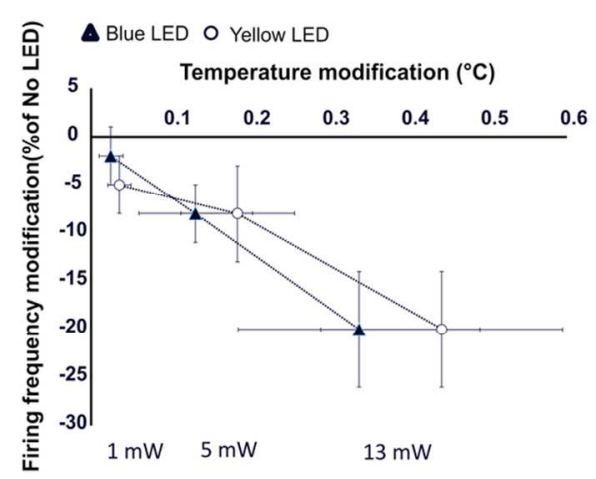

Figure 2: Tissue temperature modifications produced by LED stimulation at different powers and wavelengths. A) Example of temperature modifications induced by blue LED stimulation. B) Average temperature modification, compared to pre-light temperature, during one second stimulation with blue and yellow LEDs. p-values refer to comparisons between light and pre-light temperatures. C) Covariation of the effects of light on MC firing (data from figure 1) and slice temperature. Error bars represent $95 \%$ CI. Blue light $n=5$; yellow light $n=4$.

$99 \times 53 \mathrm{~mm}(300 \times 300$ DPI $)$ 
A
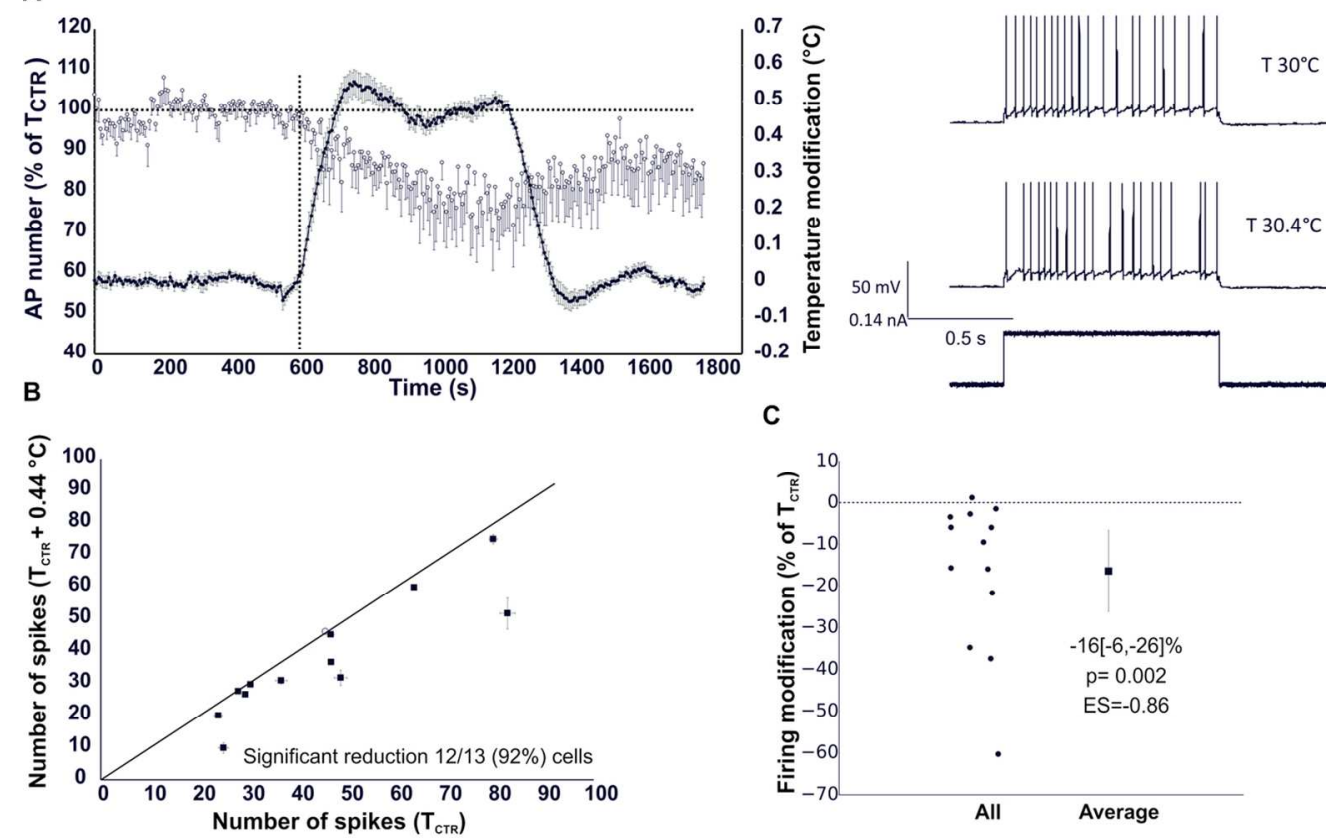

C

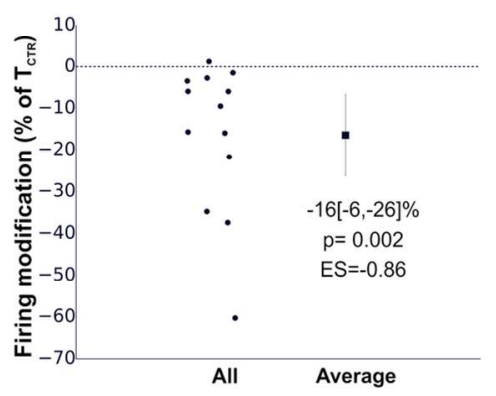

Figure 3: MC firing is reduced by temperature increase. A) Time plot of the relative modification of the number of evoked APs produced by the increase of temperature in the recording chamber. The number of APs was normalized to the average number of APs in the first 600 seconds. Error bars represent sem. Vertical dashed line represents the start time for chamber warming. Right; representative traces. B) Cellby-cell analysis of temperature effect on MC firing. Empty dots depict MCs for which no significant difference

was observed between the two conditions. Error bars represent 95\% CI. C) Population analysis of temperature effect on MC firing. Error bar represents $95 \% \mathrm{CI}$. $n=13 \mathrm{MC}$. ES=Effect Size 


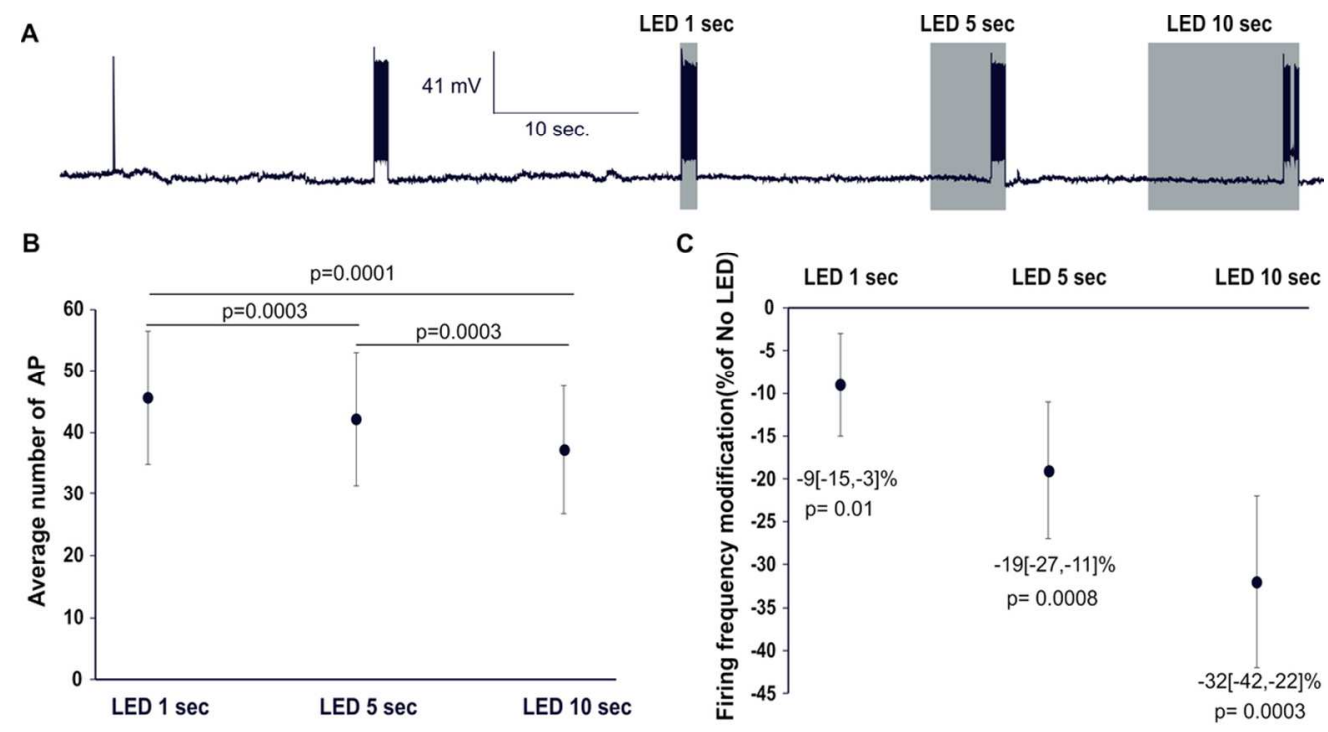

Figure 4: The effect of blue light ( $13 \mathrm{~mW}$ ) on MC firing increases with stimulus duration. A) Example of the effect of blue light stimulations on evoked MC firing; depolarizing current steps inducing firing are omitted from the figure. B) The average number of evoked action potentials decreases with increase in light duration. C) Relative modification of firing frequencies produced by light stimulation at different durations; $\mathrm{p}$-values refers to comparisons between no-LED and LED conditions. Error bars represent $95 \% \mathrm{CI}$. $\mathrm{n}=11 \mathrm{MC}$. 

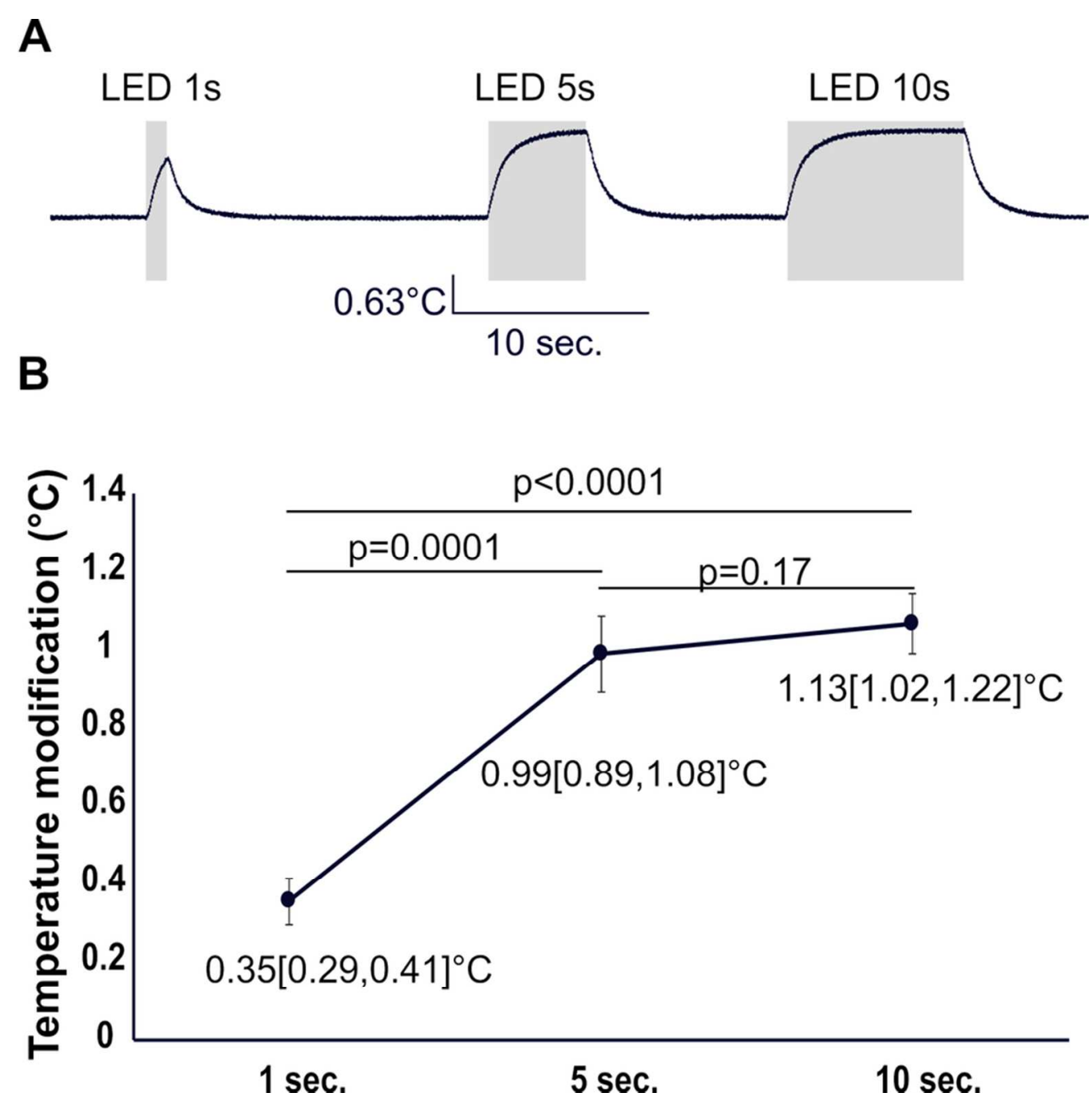

Figure 5: Modifications to slice temperatures vary with light duration. A) Example of the modification of $O B$ slice temperature produced by light stimulation (blue; $13 \mathrm{~mW}$ ) of different durations. B) Average temperature modification produced by the different durations $(n=4)$. Error bars represent $95 \%$ CI. 
A

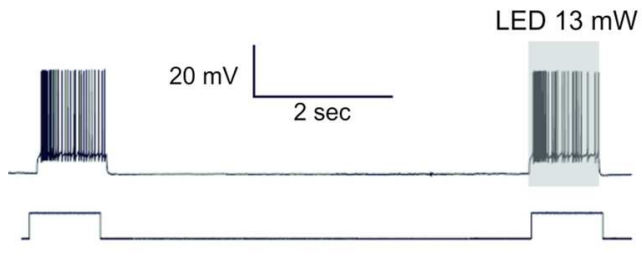

C

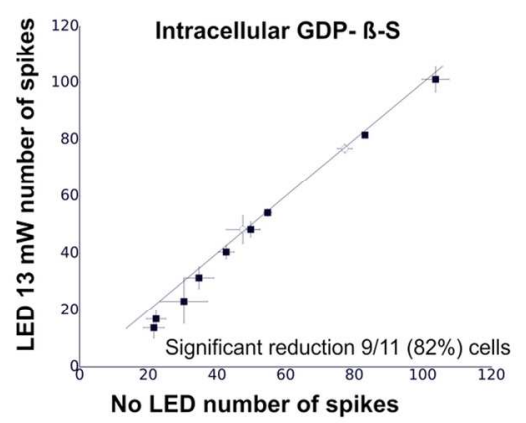

D
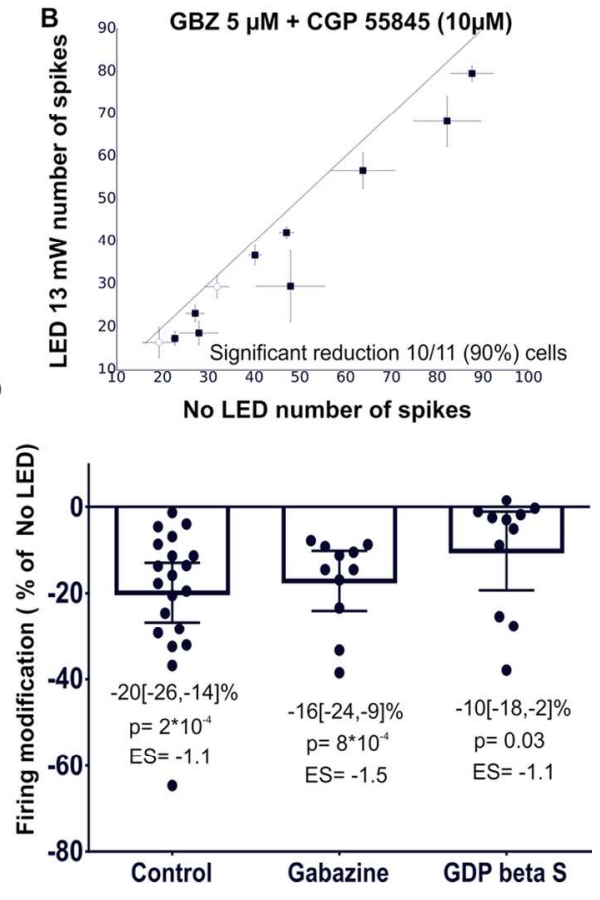

Figure 6: Activity in the inhibitory network or activation of extra-retinal photoreceptors are not required for light to modify MC firing. A) Representative example of the protocol used. B) Cell-by-cell analysis of the effect produced by LED stimulation on MC firing in the presence of GABA receptor antagonists. C) Cell-bycell analysis of the effect produced by LED stimulation on MC firing in the presence of G-protein blockers GDPßS in the intracellular solution. D) Population analysis of light-induced firing frequency modifications in the different conditions (control data are the same as figure $1 \mathrm{C}$ ). In B and C black squares depict MCs for which LED condition is significantly different compared to No-LED condition. Empty dots depict MCs for which no significant difference was observed between the two conditions. Error bars represent $95 \%$ CI. $\mathrm{n}=11 \mathrm{MC}$. ES=Effect Size

$119 \times 82 \mathrm{~mm}(300 \times 300$ DPI $)$ 


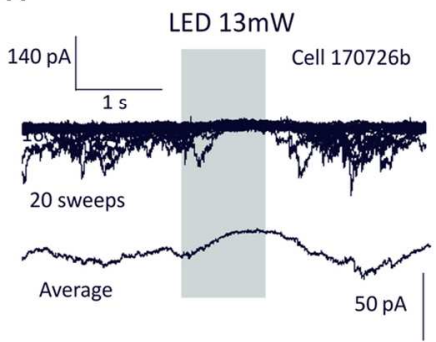

D

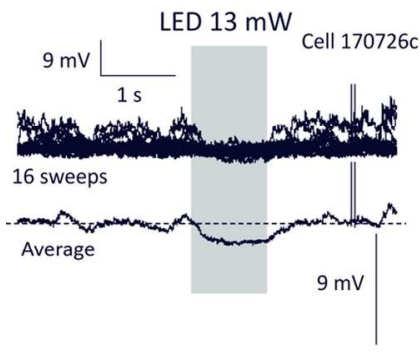

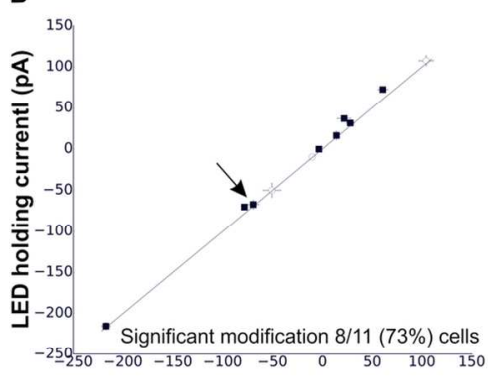

No LED holding currentl (pA)

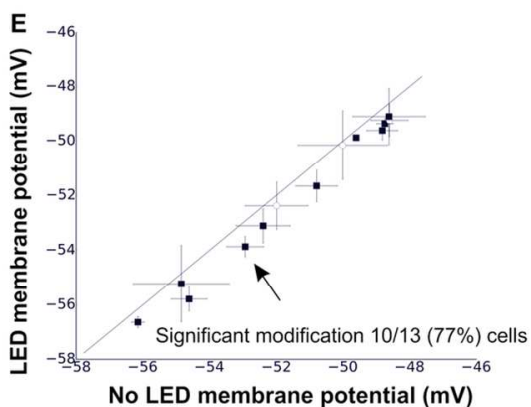

C
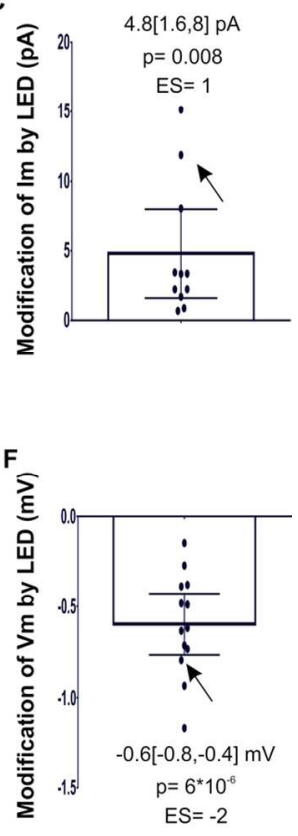

Figure 7: LED stimulation (blue light; $13 \mathrm{~mW}$ ) generates an outward current and hyperpolarizes MCs. A)

Example of light-induced outward current in one neuron. Note the reduction of the spontaneous EPSC during stimulation. B) Cell-by-cell analysis of the modification of the holding current by LED stimulation. C)

Population analysis on light- induced current modification. D) Example of light-induced modification of membrane potential in one neuron. Note the reduction of the spontaneous EPSP during stimulation. E) Cellby-cell analysis of the modification of the membrane potential by LED stimulation. F) Population analysis on light-induced modification of membrane potential. Arrows depict the analysis of the neurons represented in $A$ and D. The black squares depict MCs for which LED condition is significantly different compared to No-LED condition. Empty dots depict MCs for which no significant difference is observed between the two conditions. Error bars represent $95 \%$ CI. $n=11$ MC. ES=Effect Size 
A

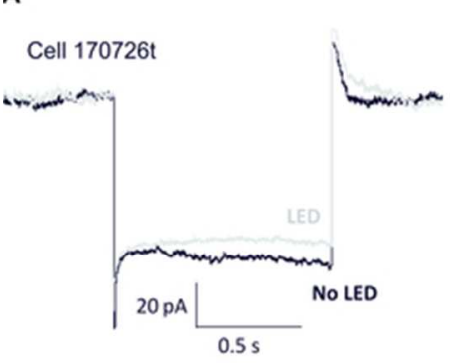

B

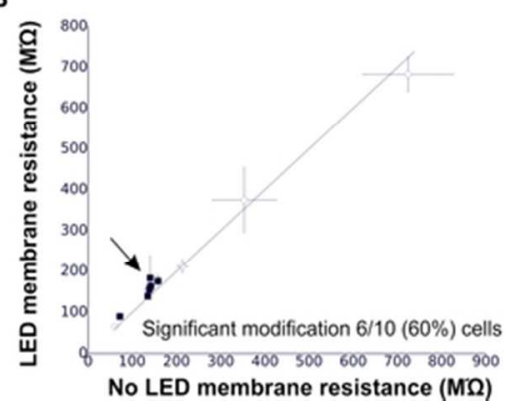

C

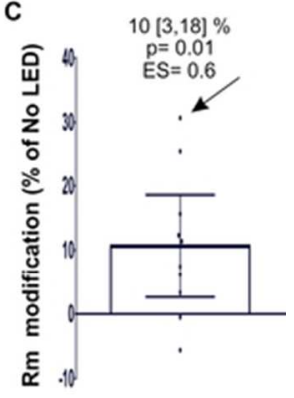

Figure 8: Light stimulation produces an apparent increase in membrane resistance in MC. A) Example of the inward currents generated by a hyperpolarizing voltage step of $10 \mathrm{mV}$ (not shown) in the presence and absence of LED stimulation (blue light; $13 \mathrm{~mW}$ ). B) Cell by cell analysis of the modification of Rm by LED stimulation. The Black squares depict MCs for which LED condition is significantly different compared to NoLED condition. Empty dots depict MCs for which no significant difference is observed between the two conditions. C) Population analysis on light induced Rm modification. Arrows depict analysis of the neuron represented in A. Error bars represent 95\% CI. $n=11$ MC. ES=Effect Size

$57 \times 18 \mathrm{~mm}(300 \times 300$ DPI $)$ 
A

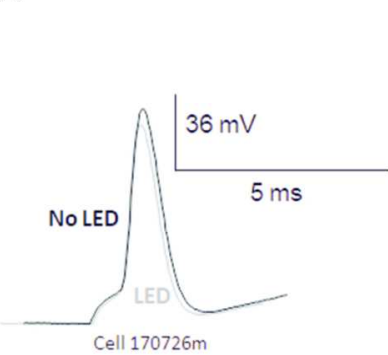

Cell $170726 \mathrm{~m}$

D

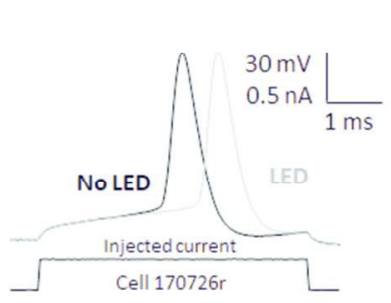

B

B

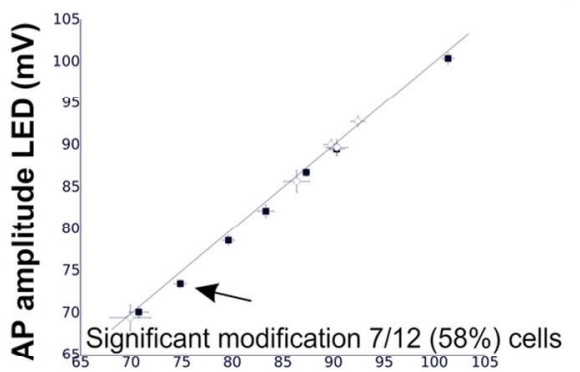

AP amplitude no LED (mV)

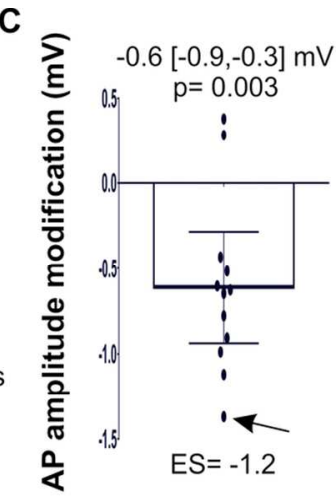

$\mathbf{F}$

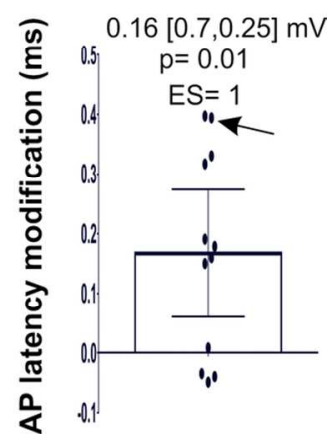

Figure 9: LED stimulation (blue light; $13 \mathrm{~mW}$ ) modify AP amplitude and latency. A) Example of effect of light on AP amplitude. The traces are aligned on action potential threshold. B) Cell-by-cell analysis of the modification of AP amplitude by LED stimulation. C) Population analysis on light-induced amplitude modification. D) Example of effect of light on AP latency. The traces are aligned vertically at the membrane potential preceding current injection. E) Cell-by-cell analysis of the modification of AP latency by LED stimulation. F) Population analysis on light-induced latency modification. In B and $E$ the black squares depict MCs for which LED condition is significantly different compared to no-LED condition. Empty dots depict MCs for which no significant difference is observed between the two conditions. Arrows depict analysis of the neurons represented in A and D. Error bars represent $95 \% \mathrm{CI}$. The $\mathrm{p}$ values are corrected for multiple comparisons (2). $n=12 \mathrm{MC}$. The $p$ values are corrected for multiple comparisons (2). ES=Effect Size 
A

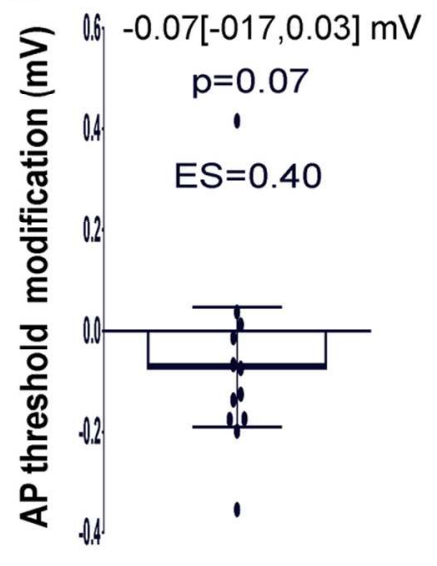

D

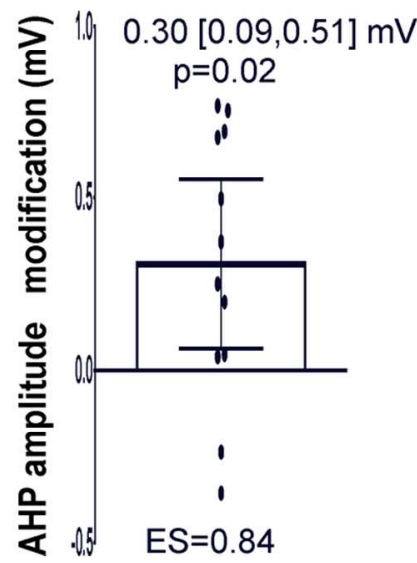

B
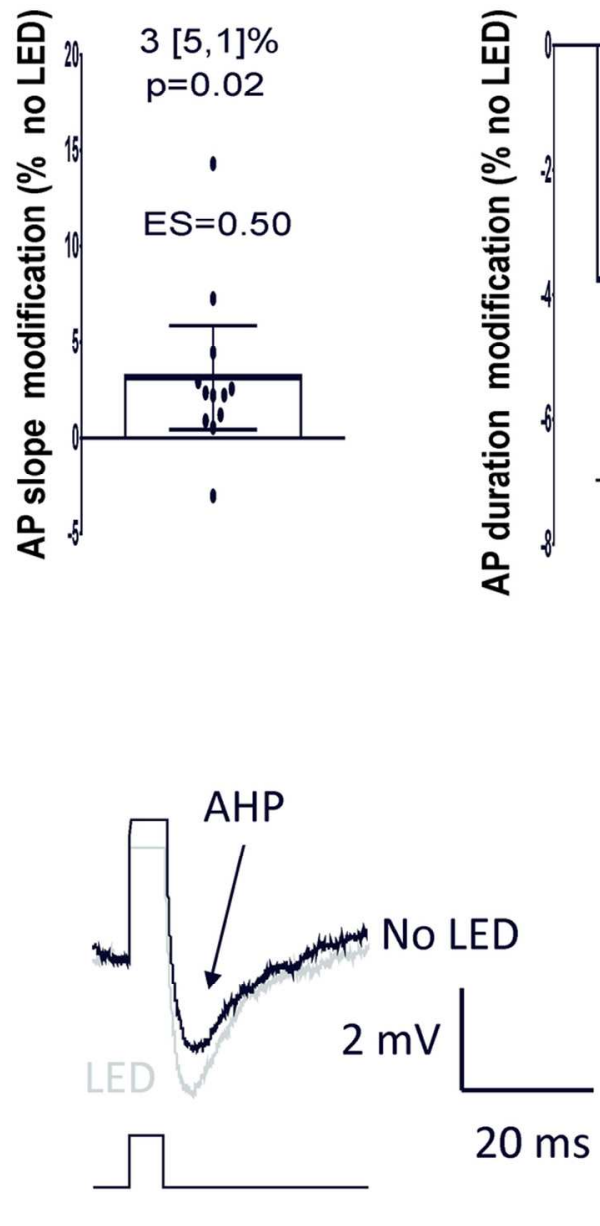

Figure 10: Exploratory analysis of LED stimulation (blue light; $13 \mathrm{~mW}$ ) on AP parameters. A) A tendency towards a decrease in AP threshold was produced by light stimulation. B) LED stimulation increases the rising slope of AP. C) Half-width duration of AP is reduced by LED stimulation D) LED stimulation produce an increase of the AHP amplitude that follows one AP; right example of the AHP in light and no-light condition. $\mathrm{n}=12 \mathrm{MC}$. The $\mathrm{p}$-values are not corrected for multiple comparisons. ES=Effect Size 


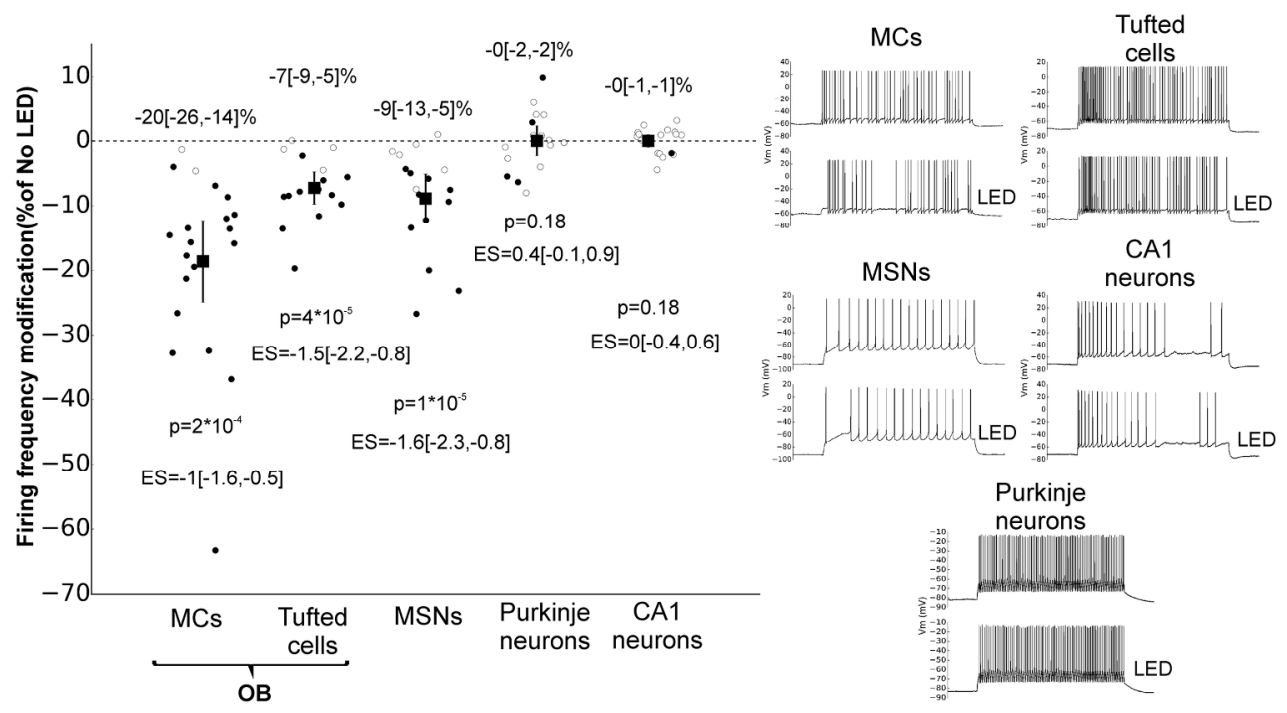

Figure 11: The effect of light on neuronal firing is dependent on neuronal type. The effect of LED stimulation (blue light; 1s starting from the beginning of the depolarizing step) on evoked firing activity is reported. $M C=$ Mitral Cells, MS= medium spiny neurons. Empty dots represent effect of light on neurons for which no statistically significant modifications were observed, black dots represent effect of light on neurons for which statistically significant modifications were observed. MC data are the same as fig $1 C(n=20)$. For tufted, MS,

Purkinje and CA1 $n=16$ and the $p$ values are corrected for multiple comparisons (4). ES=Effect Size

$104 \times 59 \mathrm{~mm}(600 \times 600 \mathrm{DPI})$ 

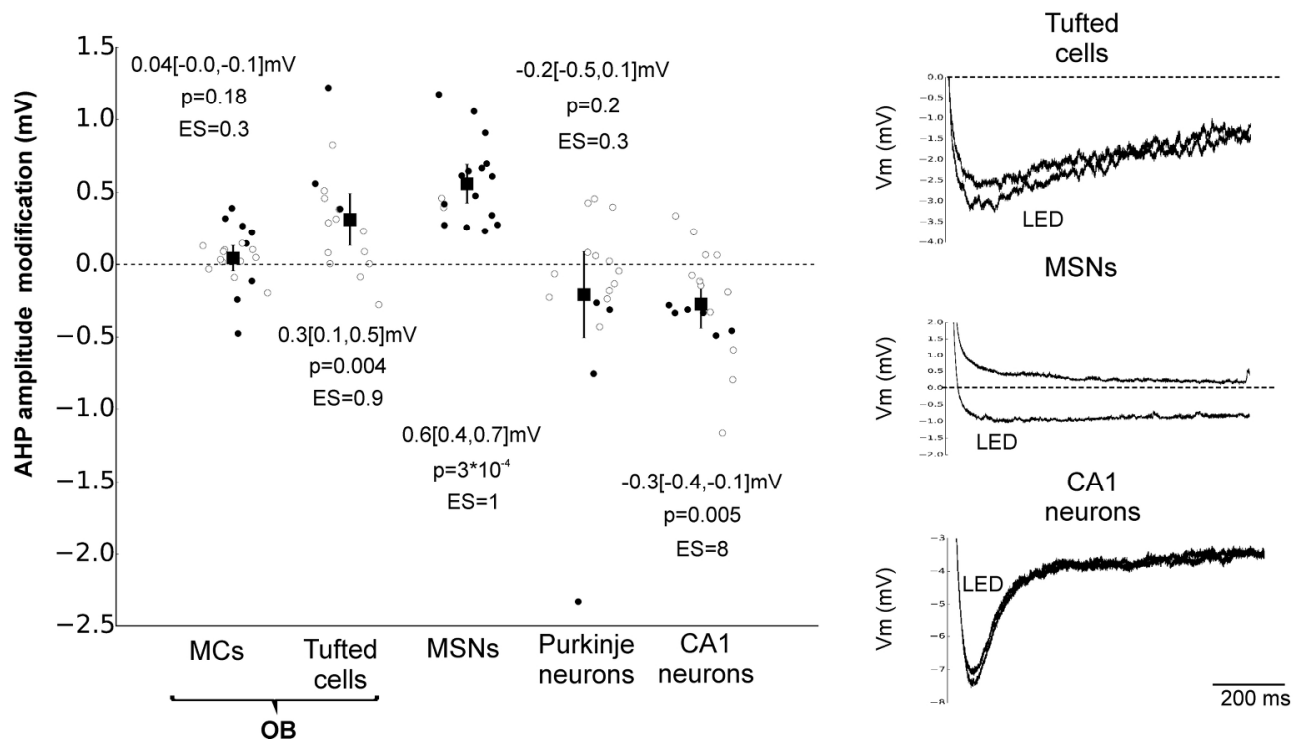

Figure 12: Exploratory analysis on effect of light on AHP amplitude. A) The effect of LED stimulation (blue light; 1s starting from the beginning of the depolarizing step) on the AHP amplitude observed at the end of the depolarization is reported. Empty dots represent effect of light on neurons for which no statistically significant modifications were observed, black dots represent effect of light on neurons for which statistically significant modifications were observed $M C=$ Mitral Cells, $M S=$ medium spiny neurons. Empty dots represent effect of light on single neurons. B) Representative trace of observed AHP modification; blue traces under LED stimulation. $M C n=20$; Tufted cells $n=16, M S N s n=17$, Purkinje neurons $n=18$ and CA1 neurons $n=18$. The $p$ values are not corrected for multiple comparisons. ES=Effect Size

$100 \times 59 m m(600 \times 600$ DPI $)$ 


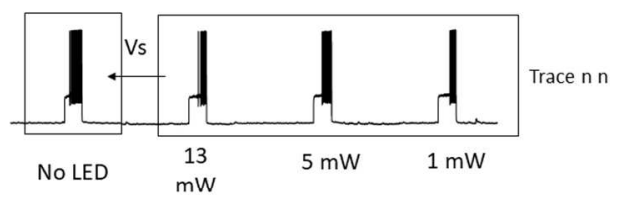

B

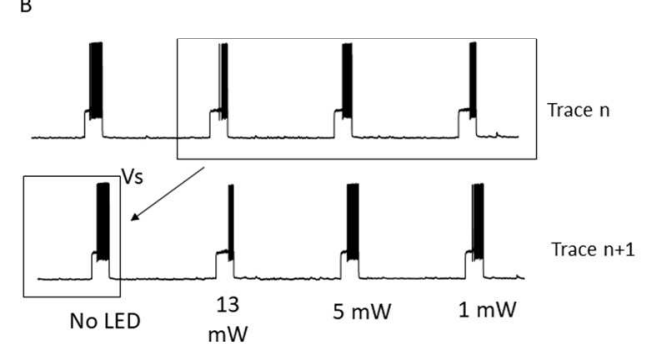

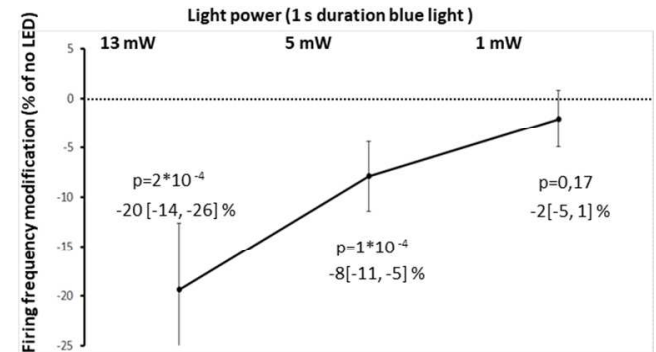

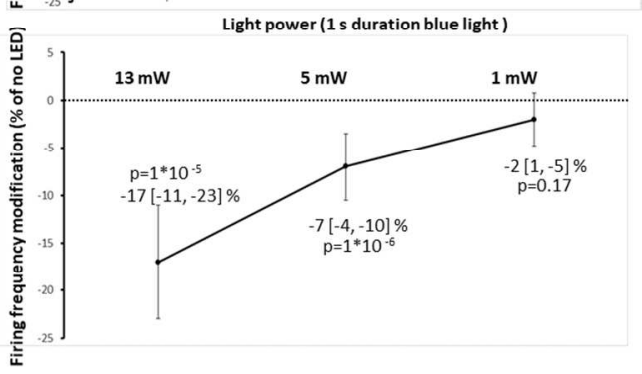

Supplementary figure 1: The reduction in firing observed during light stimulation is not due to firing rundown; A) Preregistered analysis. left; analysis procedure leading to the results presented in figure 1C. Right; analysis of the blue LED stimulation (different representation of the results illustrated in fig 1C ). B) Exploratory analysis. Shifted analysis of the same data set. Left; analysis procedure. The data were analyzed by comparing the modification of firing frequency between the LED conditions of acquisition trace $n$ and no-LED condition of acquisition trace $n+1$. Right; shifted analysis results. No qualitative difference is observed between the two types of analysis.

$338 \times 190 \mathrm{~mm}(96 \times 96 \mathrm{DPI})$ 
A

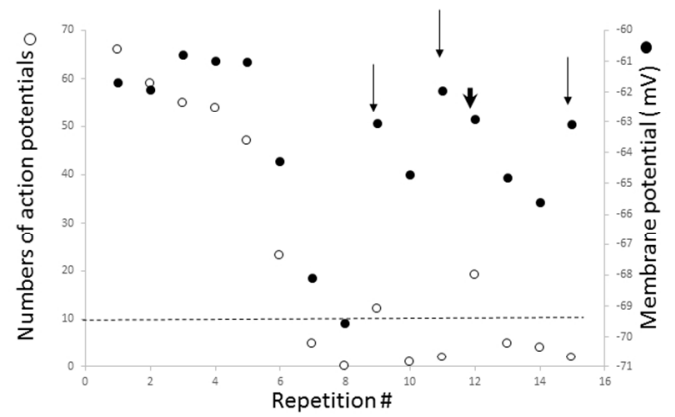

B

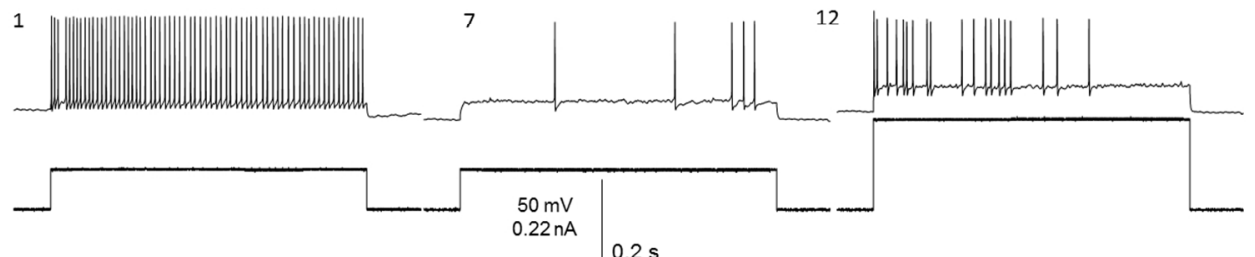

Supplementary figure 2 : The protocol used to assess whether the effect of light on MC firing depends on light duration (shown in figure 4A) producing a membrane hyperpolarization and a rundown of evoked firing activity recorded in no-LED condition. A) Typical evolution of Vrest and the number of evoked action potentials in no-LED condition. Thin arrows depict the repetitions where initial Vrest ( $-60 \mathrm{mV})$ was reset by steady positive current injection. Thick arrow depicts the repetition where the current step evoking the firing was increased in an attempt to recover the initial firing activity. Dashed line depicts the limit marking the minimal number of spikes required to include the repetition in the analysis. B) Three electrophysiological traces for no-LED condition. Up voltage membrane potential; bottom injected step current; number is the repetition. This neuron did not pass the criteria to be included in the analysis (13 repetitions having at least 10 action potentials).

$$
338 \times 190 \mathrm{~mm}(96 \times 96 \mathrm{DPI})
$$


A

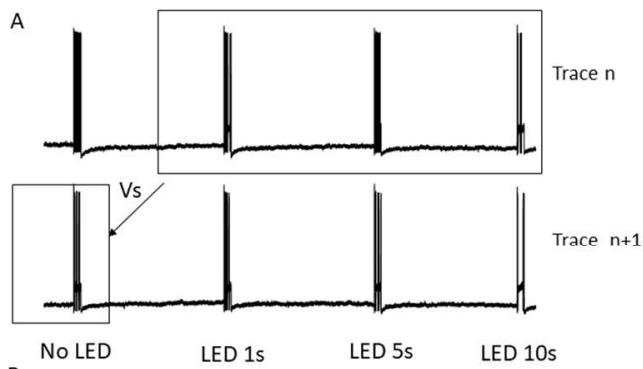

B
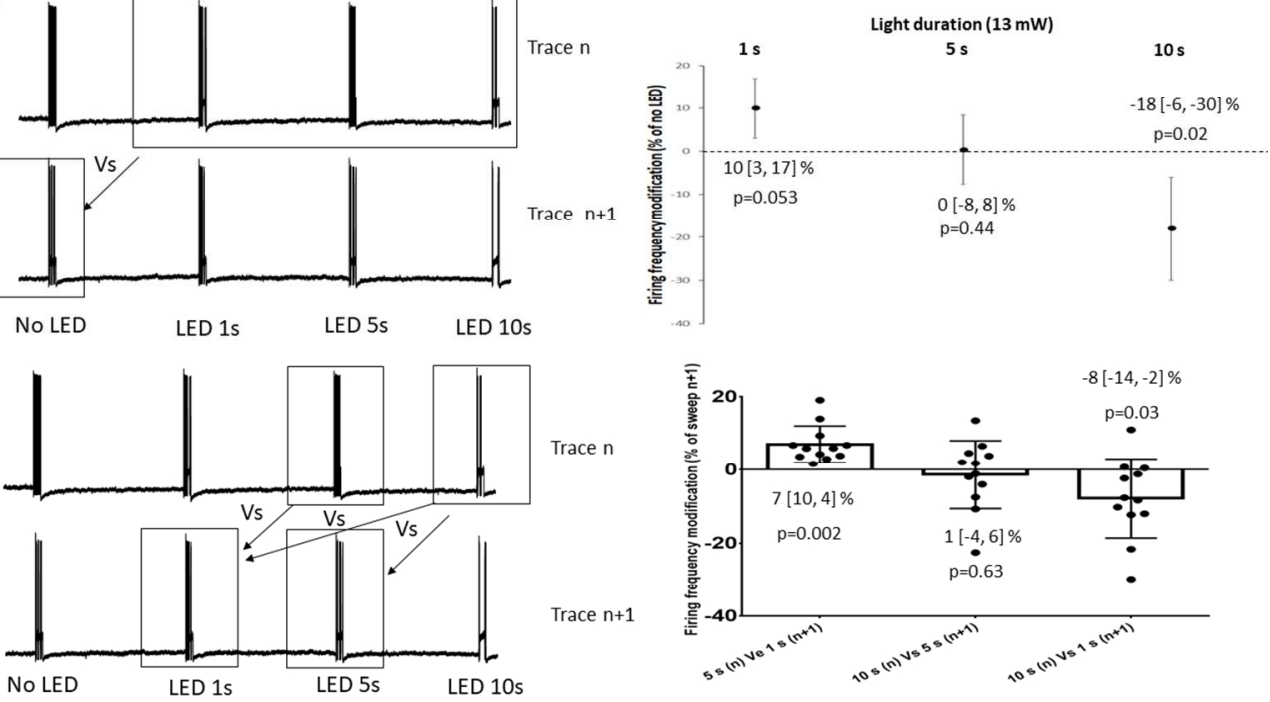

Supplementary figure 3: Firing rundown prevents the correct evaluation of the effects produced by longlasting LED stimulation. A) Left, example of shifted analysis. The data used for figure 4 were reanalyzed by comparing the modification of firing frequency between the LED conditions of acquisition trace $n$ and no-LED condition of acquisition trace $n+1$. Right, comparison of the results to those of figure 4 show that the rundown affects the capacity to evaluate the acute effect of light on neuronal firing. B) Left, shifted analysis was used to for paired comparisons between LED conditions. Right, despite rundown the firing frequency during 10-second LED stimulation of sweeps $n$ is significantly lower than the firing frequency during 1second LED stimulation of sweeps $n+1$. Error bars represent $95 \% \mathrm{CI}$. 

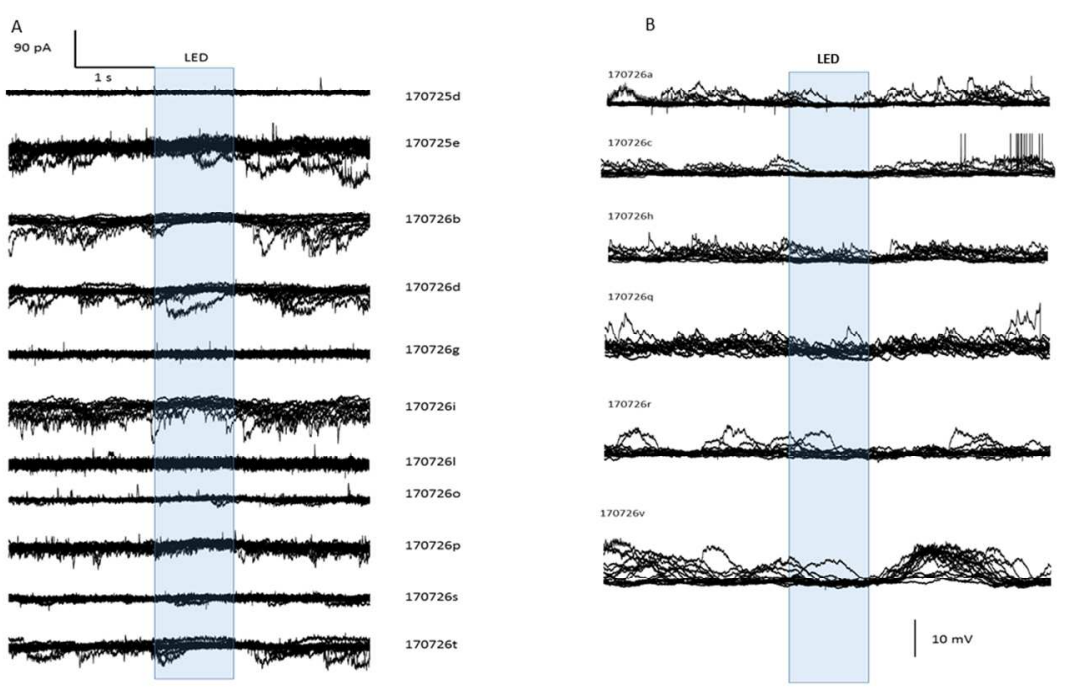

Supplementary figure 4: Effect of LED stimulation (13 mW; blue light) on: A) Holding membrane current for all recorded MCs (13-20 traces for each neuron). B) Membrane potential for MCs presenting spontaneous EPSP (13-20 traces for each neuron). Note the apparent decrease of spontaneous excitatory synaptic currents in several of the recorded MCs. 


\begin{tabular}{|c|c|c|c|c|c|c|}
\hline & \multicolumn{3}{|c|}{ Blue light } & \multicolumn{3}{c|}{ Yellow light } \\
\hline & Prior ES & Posterior ES & BF & Prior ES & Posterior ES & BF \\
\hline $\mathbf{1 3} \mathbf{m W}$ & 1.07 & 1,27 & 5635 & 1.07 & 1,28 & 6579 \\
\hline & & & & & & \\
\hline $\mathbf{5} \mathbf{m W}$ & 0,49 & 0,94 & 341 & 0,49 & 0,61 & 20 \\
\hline & & & & & & \\
\hline $\mathbf{1} \mathbf{m W}$ & 0,18 & 0,19 & 1,57 & 0,12 & 0,63 & 24 \\
\hline
\end{tabular}

Table 1: Bayesian statistic on light effect at different light power and wavelengths (bayesian one sample t-test, JASP software). Prior ES at $13 \mathrm{~mW}$ was estimated from the pilot experiment. Estimation of prior ES at $5 \mathrm{~mW}$ et $1 \mathrm{~mW}$ is calculated from the posterior ES observed respectively at $13 \mathrm{~mW}$ and $5 \mathrm{~mW}$, supposing a linear modification of the ES whit the light power, i.e. prior $\mathrm{ES}_{5 \mathrm{mw}}=\mathrm{ES}$ $13 \mathrm{mw} / 13 * 5$; prior $\mathrm{ES}_{1 \mathrm{mw}}=\mathrm{ES}_{5 \mathrm{mw}} / 5$. 
Model Comparison

\begin{tabular}{lrrrrr}
\hline \multicolumn{1}{c}{ Models } & \multicolumn{1}{c}{$\mathbf{P}(\mathbf{M}) \mathbf{P}(\mathbf{M} \mid$ data $)$} & $\mathbf{B F}_{\mathbf{M}}$ & $\mathbf{B F}_{\mathbf{1 0}}$ & error \% \\
\hline Null model (incl. subject) & 0.500 & $1.229 \mathrm{e}-6$ & $1.229 \mathrm{e}-6$ & 1.000 & \\
Temperature modification & 0.500 & 1.000 & 813869.418 & 813869.418 & 0.740 \\
\hline
\end{tabular}

Post Hoc Comparisons - Temperature modification

\begin{tabular}{lllccc}
\hline & & Prior Odds & Posterior Odds & BF $_{\mathbf{1 0}, \mathbf{U}}$ & error \% \\
\hline 1 sec. & $5 \mathrm{sec}$. & 0.587 & 91.835 & 156.342 & $6.924 \mathrm{e}-4$ \\
& $10 \mathrm{sec}$. & 0.587 & 139.777 & 237.958 & 0.001 \\
5 sec. & $10 \mathrm{sec}$. & 0.587 & 1.213 & 2.065 & $2.364 \mathrm{e}-4$ \\
\hline
\end{tabular}

Table 2: Bayesian statistic of blue light effect at different light duration (bayesian repeated measurement ANOVA, JASP software). 
Model Comparison - Light effect

\begin{tabular}{lccccr}
\hline \multicolumn{1}{c}{ Models } & $\mathbf{P}(\mathbf{M})$ & $\mathbf{P}(\mathbf{M} \mid$ data $)$ & $\mathbf{B F}_{\mathbf{M}}$ & $\mathbf{B F}_{\mathbf{1 0}}$ & \multicolumn{1}{c}{ error \% } \\
\hline Null model & 0.500 & 0.616 & 1.601 & 1.000 & \\
group & 0.500 & 0.384 & 0.624 & 0.624 & 0.031 \\
& & & & & \\
\hline
\end{tabular}

\section{Post Hoc Tests}

Post Hoc Comparisons - group

\begin{tabular}{llrrrr}
\hline & & Prior Odds & Posterior Odds & \multicolumn{1}{c}{ BF $_{\mathbf{1 0}, \mathbf{U}}$} & \multicolumn{1}{c}{ error \% } \\
\hline Ctr & GBZ +CGP & 0.587 & 0.231 & 0.393 & $2.324 \mathrm{e}-4$ \\
& GDP-beta s & 0.587 & 0.670 & 1.141 & 0.002 \\
GBZ +CGP & GDP-beta s & 0.587 & 0.429 & 0.731 & 0.008 \\
\hline
\end{tabular}

Table 3: Bayesian statistic of the effect of GABAA antagonists (GBZ and CGP) and intracellular GDPbeta s) on blue light effect (bayesian ANOVA, JASP software). 